\title{
Exact dynamics of dissipative electronic systems and quantum transport: Hierarchical equations of motion approach
}

\author{
Jinshuang Jin, Xiao Zheng, and YiJing Yan \\ Department of Chemistry, Hong Kong University of Science and Technology, Kowloon, Hong Kong
}

(Dated: 12 October 2007)

\begin{abstract}
A quantum dissipation theory is formulated in terms of hierarchically coupled equations of motion for an arbitrary electronic system coupled with grand canonical Fermion bath ensembles. The theoretical construction starts with the second-quantization influence functional in path integral formalism, in which the Fermion creation and annihilation operators are represented by Grassmann variables. Time-derivatives on influence functionals are then performed in a hierarchical manner, on the basis of calculus-on-path-integral algorithm. Both the multiple-frequency-dispersion and the non-Markovian reservoir parametrization schemes are considered for the desired hierarchy construction. The resulting formalism is in principle exact, applicable to interacting systems, with arbitrary time-dependent external fields. It renders an exact tool to evaluate various transient and stationary quantum transport properties of many-electron systems. At the second-tier truncation level the present theory recovers the real-time diagrammatic formalism developed by Schön and coworkers. For a single-particle system, the hierarchical formalism terminates at the second tier exactly, and the Landuer-Büttiker's transport current expression is readily recovered.
\end{abstract}

PACS numbers: 72.10.Bg, 05.30.-d

\section{INTRODUCTION}

The aim of this work is to establish an exact quantum dissipation theory (QDT), with which various quantum transport properties could in principle be evaluated without approximations. Quantum transport through nanosystems has conventionally been studied via the Landauer-Büttiker scattering theory $\underline{\underline{1}}^{\underline{1}}$ and nonequilibrium Green's function (NGF) formalism. 2 These approaches are however basically single-particle theories. The fundamental physics of quantum transport through an interacting system has been understood based on methods applicable to certain limits; for example, in either the weak or strong Coulomb interac-

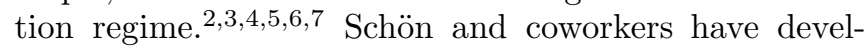
oped a formulation based on real-time diagrammatic technique $\underline{8,9,10,11,12}$ This approach could in principle be exact; however, to identify all diagrams is itself formidable, and there are practically no feasible ways to overcome this difficulty for a general non-Markovian system in the presence of arbitrary Coulomb interaction. Other promising nonperturbative methods, such as the numerical renormalization group approach, $, 3,14,15$ are yet to be extended to dynamical quantum systems.

Quantum transport is studied with the situation, where a "device", such as a semiconductor quantum dot or organic molecule under investigation, is connected to electrodes under applied bias voltages. This situation can be well described in the framework of QDT. The latter concerns the fundamental formulation that governs the dynamics of a quantum open system. The primary quantity in QDT is the reduced system density operator, $\rho(t) \equiv \operatorname{tr}_{\mathrm{B}} \rho_{\mathrm{T}}(t)$. Here, $\rho_{\mathrm{T}}(t)$ denotes the total density operator of system-bath composite; $\operatorname{tr}_{B}$ the partial trace over electrode bath degrees of freedom. Quantum transport based on QDT approach has been formu- lated extensively, $16,17,18,19,20,21,22,23$ including the aforementioned real-time diagrammatic formalism $.8,9,10,11,12$ This approach has the advantage of its generality, since different scattering processes can be handled in a unified manner, and transient dynamics can be studied readily. However, the QDTs used in quantum transport by far are all perturbative in nature; most of them are only of the second order in the system-bath coupling, 18,19,20,21,22,23 A second-order theory is also related to the sequential tunneling regime. Other limitations include the moderately high temperature/voltage and quasi-broadband (or quasi-Markovian) approximation. Quantum transport with cotunneling processes ${ }^{24.25}$ has been studied with the fourth-order QDTs, constructed via the standard

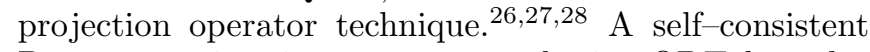
Born approximation to nonperturbative QDT has also been proposed to recover such as the nonequilibrium Kondo effect in a model system. ${ }^{23}$ Approximations involved in the existing quantum transport theories, on the basis of either QDT or NGF formalism, are subject to ever increasing challenge, due to especially the emerging fields of quantum measurement and quantum information processes $, 29,30,31,32$

This work continues our recent effort on the development of QDT formalism. ${ }^{33,34,35,36}$ The most relevant one is Ref. 36, in which a nonperturbative theory was constructed for open quantum system, interacting with Boson-like grand canonical bath ensembles. The present paper exploits the second-quantization field theory that properly treats fermionic transfer coupling processes.

The remainder of the paper is organized as follows. In Sec. III we specify the electron transfer coupling Hamiltonian and the fluctuation-dissipation theorem to be used in the later development. In Sec. IIIA we revisit the influence functional path-integral formalism, with the Fermion field representation that involves Grassmann 
variables. The derivation is detailed in Appendix A Presented in Sec. IIIB is the QDT-based expression of transient transport current. In Sec.IV] we first consider a differential form of QDT, by exploiting the calculus-onpath-integral (COPI) algorithm. 34,35,36,37,38,39 We then develop a multi-frequency-dispersed hierarchical equations of motion (MFD-HEOM) formalism of QDT. The final MFD-HEOM results are summarized in Sec. $\nabla$. The present theory is in principle exact, applicable to arbitrary quantum transport systems. It is shown in Sec.VI to recover the celebrated Landauer-Büttiker's transport current expression for single-particle systems. The involving reduced single-particle density matrix dynamics is derived in Appendix B. Moreover, it is demonstrated in Sec. VII that at the second-tier truncation the present MFD-HEOM theory recovers the real-time diagrammatic formalism developed by Schön and coworkers for Coulomb interaction systems. In Sec.VIII, we present an alternative but equivalent HEOM formalism via parametrization, with the derivation detailed in Appendix C. Finally, we conclude this work in Sec. IX]

\section{STOCHASTIC COUPLING HAMILTONIAN AND QUANTUM STATISTICAL MECHANICS}

\section{A. Stochastic transfer coupling Hamiltonian}

Consider an electron (or spin) transport setup, in which a multi-level system such as a semiconductor quantum dot or molecule is in contact with electrodes (labeled by index $\alpha$ ). Each electrode serves as an electron reservoir and is treated as a grand canonical Fermion bath ensemble. The total system-electrodes composite Hamiltonian assumes

$$
H_{\mathrm{T}}=H+\sum_{\alpha}\left(h_{\alpha}+H_{\alpha}^{\prime}\right)
$$

with the noninteracting electrons in the $\alpha$-electrode,

$$
h_{\alpha}=\sum_{k} \epsilon_{\alpha k} d_{\alpha k}^{\dagger} d_{\alpha k}
$$

and the system-electrode transfer coupling,

$$
H_{\alpha}^{\prime}=\sum_{\mu, k} t_{\alpha k \mu} d_{\alpha k}^{\dagger} a_{\mu}+\text { H.c. }
$$

H.c. stands for the Hermitian conjugate; $a_{\mu}^{\dagger}$ and $d_{\alpha k}^{\dagger}\left(a_{\mu}\right.$ and $\left.d_{\alpha k}\right)$ are the creation (annihilation) operators for an electron (or spin) in the specified spin-orbital of the system and the $\alpha$-electrode, respectively. The system Hamiltonian, $H=H\left(t ;\left\{a_{\mu}^{\dagger}, a_{\mu}\right\}\right)$ in the right-hand-side (rhs) of Eq. (2.1a), is rather arbitrary, including Coulomb interaction and coupling with external time-dependent fields such as the electric field induced by a pulsed laser.

Throughout this work, we set $\hbar \equiv 1$ and $\beta_{\alpha} \equiv$ $1 /\left(k_{\mathrm{B}} T_{\alpha}\right)$, with $k_{\mathrm{B}}$ the Boltzmann constant and $T_{\alpha}$ the temperature of $\alpha$-electrode. Electrons in bare electrode in steady state, either equilibrium or nonequilibrium, are described by the grand canonical density operator,

$$
\rho_{\alpha}^{0}=\frac{e^{-\beta_{\alpha}\left(h_{\alpha}-\mu_{\alpha} \hat{N}_{\alpha}\right)}}{\operatorname{tr}_{\mathrm{B}}\left[e^{-\beta_{\alpha}\left(h_{\alpha}-\mu_{\alpha} \hat{N}_{\alpha}\right)}\right]} .
$$

Here, $\mu_{\alpha}$ denotes the chemical potential of the $\alpha$ electrode in steady state; $\hat{N}_{\alpha}=\sum_{k} d_{\alpha k}^{\dagger} d_{\alpha k}$ is the particle number operator of electrons in $\alpha$-electrode. It satisfies $\left[\hat{N}_{\alpha}, h_{\mathrm{B}}\right]=0$, where $h_{\mathrm{B}} \equiv \sum_{\alpha} h_{\alpha}$. Denote also

$$
\langle\hat{O}\rangle_{\mathrm{B}} \equiv \operatorname{tr}_{\mathrm{B}}\left(\hat{O} \rho_{\mathrm{B}}^{0}\right) ; \quad \text { with } \quad \rho_{\mathrm{B}}^{0}=\prod_{\alpha} \rho_{\alpha}^{0} .
$$

To describe the stochastic nature of the transfer coupling, consider Eq. (2.1C) in the reservoir $h_{\mathrm{B}}$-interaction picture:

$$
H_{\alpha}^{\prime}(t)=\sum_{\mu} \hat{f}_{\alpha \mu}^{\dagger}(t) a_{\mu}+\text { H.c. },
$$

with

$$
\hat{f}_{\alpha \mu}^{\dagger}(t) \equiv e^{i h_{\mathrm{B}} t}\left[\sum_{k} t_{\alpha k \mu} d_{\alpha k}^{\dagger}\right] e^{-i h_{\mathrm{B}} t},
$$

being the stochastic interaction bath operators. They satisfy the Gaussian statistics with the Wick's theorem for thermodynamic average. Also note that $\left\langle\hat{f}_{\alpha \mu}^{\dagger}(t)\right\rangle_{\mathrm{B}}=$ $\left\langle\hat{f}_{\alpha \mu}^{\dagger}(t) \hat{f}_{\alpha \nu}^{\dagger}(\tau)\right\rangle_{\mathrm{B}}=0$, and $\left\langle\hat{f}_{\alpha \mu}^{\dagger}(t) \hat{f}_{\alpha^{\prime} \nu}(\tau)\right\rangle_{\mathrm{B}}=0$ if $\alpha \neq$ $\alpha^{\prime}$. As results, the effects of reservoirs on the reduced system can be completely determined by the two-time correlation functions,

$$
\begin{aligned}
& C_{\alpha \mu \nu}^{+}(t-\tau)=\left\langle\hat{f}_{\alpha \mu}^{\dagger}(t) \hat{f}_{\alpha \nu}(\tau)\right\rangle_{\mathrm{B}}, \\
& C_{\alpha \mu \nu}^{-}(t-\tau)=\left\langle\hat{f}_{\alpha \mu}(t) \hat{f}_{\alpha \nu}^{\dagger}(\tau)\right\rangle_{\mathrm{B}} .
\end{aligned}
$$

It follows immediately the time-reversal symmetry and the detailed-balance relations: ${ }^{36}$

$$
\left[C_{\alpha \mu \nu}^{ \pm}(t)\right]^{*}=C_{\alpha \nu \mu}^{ \pm}(-t)=e^{ \pm \beta_{\alpha} \mu_{\alpha}} C_{\alpha \mu \nu}^{\mp}\left(t-i \beta_{\alpha}\right) .
$$

Physically, $C_{\alpha \mu \nu}^{+}(t)$ describes the processes of electron tunneling from the reservoir $\alpha$ into the system, while $C_{\alpha \mu \nu}^{-}(t)$ describes the reverse events. Apparently, the reservoir correlation functions are diagonal with respect to spin indices; i.e., $C_{\alpha \mu \nu}^{ \pm}(t)=0$, if $\mu$ and $\nu$ belong to different spins. By far, the reservoir states are assumed to be time-independent. The resulting correlation functions defined in Eq. (2.6) satisfy the stationary condition of $C_{\alpha \mu \nu}^{\sigma}(t, \tau)=C_{\alpha \mu \nu}^{\sigma}(t-\tau)$. Nonstationary correlations for the case of time-dependent chemical potentials applied on electrodes will be considered in Sec. IIC see Eq. (2.16).

\section{B. Fluctuation-dissipation theorem}

For sake of bookkeeping, we shall hereafter use also $\sigma$ to label the + or - , while $\bar{\sigma} \equiv-\sigma$ the opposite sign of 
$\sigma$. Thus, Eq. (2.7) can be recast as

$$
\left[C_{\alpha \mu \nu}^{\sigma}(t)\right]^{*}=C_{\alpha \nu \mu}^{\sigma}(-t)=e^{\sigma \beta_{\alpha} \mu_{\alpha}} C_{\alpha \mu \nu}^{\bar{\sigma}}\left(t-i \beta_{\alpha}\right) .
$$

Denote also $a_{\mu}^{\sigma}$ for either the creation $\left(a_{\mu}^{+} \equiv a_{\mu}^{\dagger}\right)$ or the annihilation $\left(a_{\mu}^{-} \equiv a_{\mu}\right)$ operator for the specified spinorbital state of system.

Introduce now the spectrum functions $\Gamma_{\alpha \mu \nu}^{\sigma}(\omega)$ via

$$
C_{\alpha \mu \nu}^{\sigma}(t) \equiv \int_{-\infty}^{\infty} d \omega e^{\sigma i \omega t} \Gamma_{\alpha \mu \nu}^{\sigma}(\omega) .
$$

The above definition is consistent with the fact that the correlation functions defined in Eq. (2.6) are of $\left[C_{\alpha \mu \nu}^{+}(t)\right]^{*}=-i \Sigma_{\alpha \mu \nu}^{<}(t)$ and $C_{\alpha \mu \nu}^{-}(t)=i \Sigma_{\alpha \mu \nu}^{>}(t)$, in relation to the self-energy functions in the Green's function technique. $\stackrel{2}{\underline{2}}$ The present index scheme is however more convenient in the construction of QDT formalism. The frequency-domain counterparts of Eq. (2.8) are

$$
\Gamma_{\alpha \mu \nu}^{\sigma}(\omega)=\left[\Gamma_{\alpha \nu \mu}^{\sigma}(\omega)\right]^{*}=e^{-\sigma \beta_{\alpha}\left(\omega-\mu_{\alpha}\right)} \Gamma_{\alpha \nu \mu}^{\bar{\sigma}}(\omega) .
$$

The spectrum functions are of positivity $, 33,36$ satisfying $\Gamma_{\alpha \mu \mu}^{\sigma}(\omega) \geq 0$ and $\Gamma_{\alpha \mu \mu}^{\sigma}(\omega) \Gamma_{\alpha \nu \nu}^{\sigma}(\omega) \geq\left|\Gamma_{\alpha \mu \nu}^{\sigma}(\omega)\right|^{2}$.

To express the detailed-balance relation in terms of fluctuation-dissipation theorem (FDT), let us consider the interaction spectral density functions, defined as

$$
J_{\alpha \mu \nu}(\omega) \equiv \frac{1}{2 \pi} \int_{-\infty}^{\infty} d t e^{i \omega(t-\tau)}\left\langle\left\{\hat{f}_{\alpha \mu}(t), \hat{f}_{\alpha \nu}^{\dagger}(\tau)\right\}\right\rangle_{\mathrm{B}} .
$$

For the present model of linear coupling with noninteracting bath reservoir, it can be evaluated as $J_{\alpha \mu \nu}(\omega)=$ $\sum_{k} t_{\alpha k \mu}^{*} t_{\alpha k \nu} \delta\left(\omega-\epsilon_{\alpha k}\right)$. Together with Eqs. (2.6), (2.9), and the first identity in Eq. (2.10), we have

$$
J_{\alpha \mu \nu}(\omega)=\Gamma_{\alpha \mu \nu}^{-}(\omega)+\Gamma_{\alpha \nu \mu}^{+}(\omega)=J_{\alpha \nu \mu}^{*}(\omega) .
$$

The detailed-balance relation [i.e. the second identity in Eq. [2.10)] leads then to

$$
\begin{aligned}
& \Gamma_{\alpha \mu \nu}^{+}(\omega)=f_{\alpha}^{+}(\omega) J_{\alpha \nu \mu}(\omega), \\
& \Gamma_{\alpha \mu \nu}^{-}(\omega)=f_{\alpha}^{-}(\omega) J_{\alpha \mu \nu}(\omega),
\end{aligned}
$$

with $f_{\alpha}^{+}(\omega)=1-f_{\alpha}^{-}(\omega) \equiv f_{\alpha}(\omega)$ being the Fermi distribution function; i.e.,

$$
f_{\alpha}^{\sigma}(\omega) \equiv \frac{1}{1+e^{\sigma \beta_{\alpha}\left(\omega-\mu_{\alpha}\right)}} .
$$

By setting $J_{\alpha \mu \nu}^{+} \equiv J_{\alpha \nu \mu}$ and $J_{\alpha \mu \nu}^{-} \equiv J_{\alpha \nu \mu}$, one can write Eq. (2.13) as

$$
C_{\alpha \mu \nu}^{\sigma}(t)=\int_{-\infty}^{\infty} d \omega \frac{e^{\sigma i \omega t} J_{\alpha \mu \nu}^{\sigma}(\omega)}{1+e^{\sigma \beta_{\alpha}\left(\omega-\mu_{\alpha}\right)}} .
$$

This is the FDT in the fermionic grand canonical ensembles. It relates the correlation functions to the spectral densities.

\section{Correlation functions in the presence of time-dependent chemical potentials}

We shall also be interested in the transient dynamics of charge transport under time-dependent bias voltage. Its effect can be described by rigid homogeneous timedependent shifts of the conduction bands of electrodes; i.e., $\epsilon_{\alpha k \mu}(t)=\epsilon_{\alpha k \mu}+\Delta_{\alpha}(t)$ and $\mu_{\alpha}(t)=\mu_{\alpha}+\Delta_{\alpha}(t)$, so that the occupation on each state is unchanged. 22 As results, the nonstationary correlation functions are

$$
C_{\alpha \mu \nu}^{\sigma}(t, \tau)=\exp \left[\sigma i \int_{\tau}^{t} d t^{\prime} \Delta_{\alpha}\left(t^{\prime}\right)\right] C_{\alpha \mu \nu}^{\sigma}(t-\tau),
$$

for $t \geq \tau$. Note that $\Delta_{\alpha}(t)$ in this work represents timedependent chemical potential, on top of the constant part $\left(\mu_{\alpha}-\mu_{\alpha}^{\mathrm{eq}}\right)$ applied on $\alpha$-electrode.

\section{QUANTUM TRANSPORT VERSUS DISSIPATION}

\section{A. Influence functional in path integral formalism}

We shall be interested in a QDT-based transport formulation. Let us first revisit the path-integral (PI) influence functional expression. It serves as the starting point for the development of HEOM formalism of QDT $34,35,36,37,38,39$ The quantity of interest here is the reduced density operator, defined as the trace of the total density operator over the bath subspace; i.e., $\rho(t) \equiv$ $\operatorname{tr}_{\mathrm{B}} \rho_{\mathrm{T}}(t)$. The quantum dissipation starts with the initial condition that the system and bath were initially uncorrelated:

$$
\rho_{\mathrm{T}}\left(t_{0}\right)=\rho\left(t_{0}\right) \rho_{\mathrm{B}}^{0} ; \quad t_{0} \rightarrow-\infty .
$$

This initial factorization ansatz does not cause approximation, as long as the initial time is set to infinite past $33,40,41$ We will come back to this issue in Sec. IX.

The key variation from our previous work with Bosonlike reservoir ${ }^{36}$ is the second quantization that leads to the Grassmann variables for Fermion fields rather than the ordinary c-numbers in the PI representation. $\stackrel{42.43}{=}$ To proceed, let $\{|\psi\rangle\}$ be a second-quantization basis set in the system subspace, and $\boldsymbol{\psi} \equiv\left(\psi, \psi^{\prime}\right)$ for short, so that $\rho(\boldsymbol{\psi}, t) \equiv \rho\left(\psi, \psi^{\prime}, t\right)$. Denote $\mathcal{U}\left(t, t_{0}\right)$ as the reduced Liouville-space propagator, by which

$$
\rho(t) \equiv \mathcal{U}\left(t, t_{0}\right) \rho\left(t_{0}\right) .
$$

Its $\mathrm{PI}$ expression in the $\psi$-representation reads $\underline{44}$

$$
\mathcal{U}\left(\boldsymbol{\psi}, t ; \boldsymbol{\psi}_{0}, t_{0}\right)=\int_{\boldsymbol{\psi}_{0}\left[t_{0}\right]}^{\boldsymbol{\psi}[t]} \mathcal{D} \boldsymbol{\psi} e^{i S[\psi]} \mathcal{F}[\boldsymbol{\psi}] e^{-i S\left[\psi^{\prime}\right]} .
$$

$S[\psi]$ is the classical action functional of the reduced system, evaluated along a path $\psi(\tau)$, with the constraints that two ending points $\psi\left(t_{0}\right)=\psi_{0}$ and $\psi(t)=\psi$ are fixed. 
The key quantity in PI expression is the influence functional, $\mathcal{F}$ in Eq. 3.2b). For the present model of linear coupling with noninteracting electron reservoir, it can be formally evaluated by using the Wick's theorem for the thermodynamic Gaussian average, implemented with Grassmann algebra. ${ }^{42,43}$ The final results read [cf. Eq. (A6) ]

$$
\mathcal{F}[\boldsymbol{\psi}]=\exp \left\{-\int_{t_{0}}^{t} d \tau \mathcal{R}[\tau ;\{\boldsymbol{\psi}\}]\right\}
$$

with (denoting $a_{\mu}^{+} \equiv a_{\mu}^{\dagger}$ and $a_{\mu}^{-} \equiv a_{\mu}$ )

$$
\mathcal{R}[t ;\{\boldsymbol{\psi}\}] \equiv i \sum_{\alpha, \mu, \sigma} \mathcal{A}_{\mu}^{\bar{\sigma}}[\boldsymbol{\psi}(t)] \mathcal{B}_{\alpha \mu}^{\sigma}(t ;\{\boldsymbol{\psi}\})
$$

Here, $\mathcal{A}_{\mu}^{\sigma}$ and $\mathcal{B}_{\alpha \mu}^{\sigma}$ are Grassmann variables, defined as

$$
\mathcal{A}_{\mu}^{\sigma}[\boldsymbol{\psi}(t)] \equiv a_{\mu}^{\sigma}[\psi(t)]+a_{\mu}^{\sigma}\left[\psi^{\prime}(t)\right]
$$

and

$$
\mathcal{B}_{\alpha \mu}^{\sigma}(t ;\{\boldsymbol{\psi}\}) \equiv-i\left[B_{\alpha \mu}^{\sigma}(t ;\{\psi\})-B_{\alpha \mu}^{\prime \sigma}\left(t ;\left\{\psi^{\prime}\right\}\right)\right]
$$

with

$$
\begin{aligned}
B_{\alpha \mu}^{\sigma}(t ;\{\psi\}) & \equiv \sum_{\nu} \int_{t_{0}}^{t} d \tau C_{\alpha \mu \nu}^{\sigma}(t, \tau) a_{\nu}^{\sigma}[\psi(\tau)] \\
B_{\alpha \mu}^{\prime \sigma}\left(t ;\left\{\psi^{\prime}\right\}\right) & \equiv \sum_{\nu} \int_{t_{0}}^{t} d \tau C_{\alpha \mu \nu}^{\bar{\sigma} *}(t, \tau) a_{\nu}^{\sigma}\left[\psi^{\prime}(\tau)\right]
\end{aligned}
$$

Note that $B_{\alpha \mu}^{\prime \sigma}=\left(B_{\alpha \mu}^{\bar{\sigma}}\right)^{\dagger}$ leads to $\mathcal{B}_{\alpha \mu}^{\bar{\sigma}}=\left(\mathcal{B}_{\alpha \mu}^{\sigma}\right)^{\dagger}$. This property corresponds to the Hermitian conjugate relation of auxiliary density operators that will be discussed later; see Eqs. (3.12), (5.5) or (8.8). The interaction reservoir correlation functions in Eq. (3.6) can be nonstationary to support the study of transient current under timedependent bias voltage applied to electrodes.

All variables involved in Eqs. (3.4) and (3.5) stem from second-quantization of fermion operators. They are Grassmann variables in PI formalism,,$\frac{42}{,}$ following the anticommutation relation, such as $\mathcal{B}_{\mu}^{\sigma} a_{\alpha \mu}^{\bar{\sigma}}=-a_{\mu}^{\bar{\sigma}} \mathcal{B}_{\alpha \mu}^{\sigma}$. Apparently, the dissipation functional $\mathcal{R}$ [Eq. (3.3b)], as it consists of bi-fermionic variables, and the influence functional $\mathcal{F}$ [Eq. (3.3a)] remain as ordinary c-numbers.

The time derivative on $\mathcal{F}$ reads

$$
\partial_{t} \mathcal{F}=-\mathcal{R} \mathcal{F}=-i \sum_{\alpha, \mu, \sigma} \mathcal{A}_{\mu}^{\bar{\sigma}} \mathcal{B}_{\alpha \mu}^{\sigma} \mathcal{F} \equiv-i \sum_{\alpha, \mu, \sigma} \mathcal{A}_{\mu}^{\bar{\sigma}} \mathcal{F}_{\alpha \mu}^{\sigma}
$$

Introduce here are a set of first-tier auxiliary influence functionals (AIFs),

$$
\mathcal{F}_{\alpha \mu}^{\sigma} \equiv \mathcal{B}_{\alpha \mu}^{\sigma} \mathcal{F}
$$

They are Grassmann variables, for which [cf. Eq. (3.4)]

$$
\mathcal{A}_{\mu}^{\bar{\sigma}} \mathcal{F}_{\alpha \mu}^{\sigma}=a_{\mu}^{\bar{\sigma}}[\psi(t)] \mathcal{F}_{\alpha \mu}^{\sigma}-\mathcal{F}_{\alpha \mu}^{\sigma} a_{\mu}^{\bar{\sigma}}\left[\psi^{\prime}(t)\right] .
$$

The identity of $a_{\mu}^{\bar{\sigma}}\left[\psi^{\prime}(t)\right] \mathcal{F}_{\alpha \mu}^{\sigma}=-\mathcal{F}_{\alpha \mu}^{\sigma} a_{\mu}^{\bar{\sigma}}\left[\psi^{\prime}(t)\right]$ for Grassmann variables is used here.

The first-tier auxiliary density operators (ADOs) can now be defined via [cf. Eq. (3.2)]

$$
\rho_{\alpha \mu}^{\sigma}(t) \equiv \mathcal{U}_{\alpha \mu}^{\sigma}\left(t, t_{0}\right) \rho\left(t_{0}\right)
$$

with

$$
\mathcal{U}_{\alpha \mu}^{\sigma}\left(\boldsymbol{\psi}, t ; \boldsymbol{\psi}_{0}, t_{0}\right) \equiv \int_{\boldsymbol{\psi}_{0}\left[t_{0}\right]}^{\boldsymbol{\psi}[t]} \mathcal{D} \boldsymbol{\psi} e^{i S[\psi]} \mathcal{F}_{\alpha \mu}^{\sigma}[\boldsymbol{\psi}] e^{-i S\left[\psi^{\prime}\right]}
$$

We can then recast Eq. (3.7) in terms of the reduced density operator and its auxiliary ones as

$$
\dot{\rho}(t)=-i \mathcal{L} \rho(t)-i \sum_{\alpha, \mu, \sigma}\left[a_{\mu}^{\bar{\sigma}}, \rho_{\alpha \mu}^{\sigma}(t)\right]
$$

The first term in which $\mathcal{L} \rho \equiv[H, \rho]$ arises from the time derivative of classical action exponential term of Eq. (3.2). The commutator in the second term can be recast as $\left[a^{\bar{\sigma}}, \rho_{\alpha \mu}^{\sigma}\right]=\mathcal{A}_{\mu}^{\bar{\sigma}} \rho_{\alpha \mu}^{\sigma}$ that is the operator-level counterpart of Eq. (3.9). The Hermitian conjugate relation implied in Eq. (3.5) reads

$$
\rho_{\alpha \mu}^{\bar{\sigma}}=\left(\rho_{\alpha \mu}^{\sigma}\right)^{\dagger}
$$

This identity can also be verified via the equivalent operator-level definition of the first-tier $\operatorname{ADO} \rho_{\alpha \mu}^{\sigma}$; see Eq. (3.16).

\section{B. Quantum transport current via the auxiliary reduced density operator dynamics}

In this subsection, we shall show that the transport current is directly related to the first-tier ADOs. The final expression for the transient current from $\alpha$-lead to system reads (setting $e=1$ )

$$
I_{\alpha}(t)=i \sum_{\mu} \operatorname{tr}_{\mathrm{S}}\left\{\rho_{\alpha \mu}^{+}(t) a_{\mu}-a_{\mu}^{\dagger} \rho_{\alpha \mu}^{-}(t)\right\} .
$$

Here, $\rho_{\alpha \mu}^{+}=\left(\rho_{\alpha \mu}^{-}\right)^{\dagger}$ are the aforementioned first-tier ADOs. The total current passing through the system from $\mathrm{L}$ to $\mathrm{R}$ electrode is then $I(t)=I_{\mathrm{L}}(t)-I_{\mathrm{R}}(t)$.

We are now in the position to prove the current expression, Eq. (3.13). Let us start with the definition,

$$
I_{\alpha}(t)=-\frac{d}{d t}\left\langle\hat{N}_{\alpha}\right\rangle=i\left\langle\left[\hat{N}_{\alpha}, H_{\mathrm{T}}(t)\right]\right\rangle_{\mathrm{T}} .
$$

Here, $\langle\cdot\rangle_{\mathrm{T}}=\operatorname{Tr}_{\mathrm{T}}\left[\cdot \rho_{\mathrm{T}}(t)\right]$, with $\operatorname{Tr}_{\mathrm{T}} \equiv \operatorname{tr}_{\mathrm{S}} \operatorname{tr}_{\mathrm{B}} ; \hat{N}_{\alpha}$ is the electron number operator in the electrode $\alpha$, satisfying $\left[\hat{N}_{\alpha}, H(t)\right]=\left[\hat{N}_{\alpha}, h_{\mathrm{B}}\right]=0 . \quad$ In Eq. (3.14),$H_{\mathrm{T}}=H+$ $H^{\prime}(t)$ is the total composite Hamiltonian given in the $h_{\mathrm{B}}$-interaction picture. One can readily obtain

$$
I_{\alpha}(t)=i \sum_{\mu}\left\langle\left[\hat{f}_{\alpha \mu}^{\dagger}(t) a_{\mu}-a_{\mu}^{\dagger} \hat{f}_{\alpha \mu}(t)\right]\right\rangle_{\mathrm{T}} .
$$


The equivalence between Eq. (3.14) and Eq. (3.15) can be established by recognizing the fact that the first-tier ADOs defined in the previous subsection can be recast as

$$
\rho_{\alpha \mu}^{\sigma}(t)=\operatorname{tr}_{\mathrm{B}}\left[\hat{f}_{\alpha \mu}^{\sigma}(t) \rho_{\mathrm{T}}(t)\right]
$$

In fact $\dot{\rho}_{\mathrm{T}}(t)=-i\left[H_{\mathrm{T}}(t), \rho_{\mathrm{T}}(t)\right]$ in this work reads [cf. Eq. (2.4)]

$$
\dot{\rho}_{\mathrm{T}}(t)=-i \mathcal{L} \rho_{\mathrm{T}}(t)-i \sum_{\alpha \mu}\left[\hat{f}_{\alpha \mu}^{\dagger}(t) a_{\mu}+a_{\mu}^{\dagger} \hat{f}_{\alpha \mu}(t), \rho_{\mathrm{T}}(t)\right] .
$$

Applying now the definition of $\rho(t) \equiv \operatorname{tr}_{\mathrm{B}} \rho_{\mathrm{T}}(t)$, the trace cyclic invariance, and Eq. (3.11), it concludes immediately that Eq. (3.16) does amount to the first-tier ADO defined earlier. This equivalence can also be proved by the explicit PI expression of Eq. (3.16).

We have therefore established the transport current expression [Eq. (3.13)], in terms of the reduced density operator, more precisely the first-tier ADO dynamics. Note that the charge current in Eq. (3.13) is of the form of $I_{\alpha}(t)=\sum_{\mu} I_{\alpha \mu}(t)$. It consists of the contributions from individual spin-orbitals that couple directly with the lead. Physically, it is useful to study individual contributions, for example spin-dependent current.

\section{HIERARCHICAL EQUATIONS OF MOTION APPROACH TO QUANTUM DISSIPATION}

To complete the formulation, we shall develop hierarchically coupled EOM for evaluating both $\rho$ and its ADOs, in the presence of arbitrary non-Markovian dissipation. Both MFD and parametrization schemes of hierarchy will be considered. The desired theory will be constructed via the calculus-on-path-integral (COPI) algorithm. ${ }^{34,35,36,37,38,39}$ For clarity, we present in this section the derivation of MFD-HEOM formalism, while summarize its final results in the next section. The parametrization based HEOM formalism will be postponed to Sec.VIII after its MFD equivalence is scrutinized in relation with the Landauer-Büttiker's and the real-time diagrammatic formulations of quantum transport.

\section{A. Quantum dissipation theory via calculus on path integrals}

In the following development we omit the explicit PI variables dependence whenever it does not cause confusion. Adopt further the abbreviation $j=\{\mu \sigma\}$ or $\{\alpha \mu \sigma\}$ whenever applicable. So that $\mathcal{A}_{\bar{j}} \equiv \mathcal{A}_{\mu}^{\bar{\sigma}}, \mathcal{B}_{j} \equiv \mathcal{B}_{\alpha \mu}^{\sigma}$, and Eq. (3.7) is written as

$$
\partial_{t} \mathcal{F}=-i \sum_{j} \mathcal{A}_{\bar{j}} \mathcal{B}_{j} \mathcal{F}
$$

Note that $\mathcal{A}_{j}$ [Eq. (3.4)] depends on the field at the fixed ending point $\psi(t)=\psi$ of the path. The difficulty of the PI formalism is the evaluation of $\mathcal{B}_{j}$ [Eq. (3.5)] that involves $\psi(\tau)$ at all $t_{0} \leq \tau \leq t$. To resolve the memory contained in $\mathcal{B}_{j}$, consider its time derivative,

$$
\partial_{t} \mathcal{B}_{j}=\tilde{\mathcal{B}}_{j}-i \tilde{\mathcal{C}_{j}}
$$

Here,

$$
\tilde{\mathcal{B}}_{j} \equiv \tilde{\mathcal{B}}_{\alpha \mu}^{\sigma}=-i\left[\tilde{B}_{\alpha \mu}^{\sigma}-\tilde{B}_{\alpha \mu}^{\prime \sigma}\right],
$$

that is similar to $\mathcal{B}_{j}$, but with [cf. Eqs. (3.5) and (3.6)]

$$
\tilde{B}_{\alpha \mu}^{\sigma}(t ;\{\psi\})=\sum_{\nu} \int_{t_{0}}^{t} d \tau \dot{C}_{\alpha \mu \nu}^{\sigma}(t, \tau) a_{\nu}^{\sigma}[\psi(\tau)],
$$

and [denoting $C_{\alpha \mu \nu}^{0, \sigma} \equiv C_{\alpha \mu \nu}^{\sigma}(0)=C_{\alpha \nu \mu}^{\sigma *}(0)$; cf. Eq. (2.7)]

$$
\tilde{\mathcal{C}}_{j} \equiv \sum_{\nu}\left\{C_{\alpha \mu \nu}^{0, \sigma} a_{\nu}^{\sigma}[\psi(t)]-C_{\alpha \nu \mu}^{0, \bar{\sigma}} a_{\nu}^{\sigma}\left[\psi^{\prime}(t)\right]\right\} .
$$

Note that $C_{\alpha \mu \nu}^{\sigma}(t, t)=C_{\alpha \mu \nu}^{\sigma}(0)$ used in Eq. (4.4) is valid for either stationary or nonstationary reservoir correlation functions. Apparently, both $\tilde{\mathcal{B}}_{j}$ and $\tilde{\mathcal{C}}_{j}$ are Grassmann variables. The former contains memory, while the latter depends only on the fixed ending point of the path.

To continue the hierarchy construction, consider now the time-derivative on the first-tier AIF of Eq. (3.8). Using Eqs. (4.1) and (4.2), we have (noting that $\mathcal{F}_{j} \equiv \mathcal{F}_{\alpha \mu}^{\sigma}$ )

$$
\begin{aligned}
\partial_{t} \mathcal{F}_{j} & \equiv \partial_{t}\left(\mathcal{B}_{j} \mathcal{F}\right) \\
& =\left(\tilde{\mathcal{B}}_{j}-i \tilde{\mathcal{C}}_{j}\right) \mathcal{F}-i \sum_{j^{\prime}} \mathcal{B}_{j} \mathcal{A}_{\bar{j}^{\prime}} \mathcal{B}_{j^{\prime}} \mathcal{F} \\
& =\tilde{\mathcal{B}}_{j} \mathcal{F}-i \tilde{\mathcal{C}}_{j} \mathcal{F}-i \sum_{j^{\prime}} \mathcal{A}_{\bar{j}^{\prime}} \mathcal{B}_{j^{\prime}} \mathcal{B}_{j} \mathcal{F} \\
& \equiv \tilde{\mathcal{F}}_{j}-i \tilde{\mathcal{C}}_{j} \mathcal{F}-i \sum_{j^{\prime}} \mathcal{A}_{\bar{j}^{\prime}} \mathcal{F}_{j j^{\prime}} .
\end{aligned}
$$

Note that $\tilde{\mathcal{F}}_{j} \equiv \tilde{\mathcal{B}}_{j} \mathcal{F}$, which is a Grassmann variable, belongs to a different class of first-tier AIF. We shall return to this issue in the next subsection.

The last term in Eq. (4.5) defines also the second-tier AIFs; i.e.,

$$
\mathcal{F}_{j j^{\prime}} \equiv \mathcal{B}_{j^{\prime}} \mathcal{B}_{j} \mathcal{F}=-\mathcal{F}_{j^{\prime} j} .
$$

The second identity that implies $\mathcal{F}_{j j}=0$ arises from the Grassmann anticommutation relation of $\mathcal{B}_{j^{\prime}} \mathcal{B}_{j}=$ $-\mathcal{B}_{j} \mathcal{B}_{j^{\prime}}$

The second-tier AIFs are ordinary c-numbers. Thus, $\mathcal{A}_{\bar{j}^{\prime}} \mathcal{F}_{j j^{\prime}}$ in the last term of Eq. (4.5) amounts to [noting that $\mathcal{A}_{\bar{j}^{\prime}} \equiv \mathcal{A}_{\mu^{\prime}}^{\bar{\sigma}^{\prime}}$ of Eq. [3.4)]

$$
\mathcal{A}_{\bar{j}^{\prime}} \mathcal{F}_{j j^{\prime}}=a_{\mu^{\prime}}^{\bar{\sigma}{ }^{\prime}}[\psi(t)] \mathcal{F}_{j j^{\prime}}+\mathcal{F}_{j j^{\prime}} a_{\mu^{\prime}}^{\bar{\sigma}^{\prime}}\left[\psi^{\prime}(t)\right] .
$$

It is in contrast to Eq. (3.9), which involves a first-tier AIF that is a Grassmann variable. 
We are now in the position to summarize the above hierarchy construction. An $n^{\text {th }}$-tier AIFs can be defined in general as

$$
\mathcal{F}_{\mathbf{j}}^{(n)} \equiv \mathcal{F}_{j_{1} j_{2} \cdots j_{n}} \equiv \mathcal{B}_{j_{n}} \cdots \mathcal{B}_{j_{2}} \mathcal{B}_{j_{1}} \mathcal{F} .
$$

Note that exchanging any two indexes in a given AIF companies with a "-" sign. This leads to the property that the $n$ indexes in $\mathcal{F}_{j_{1} j_{2} \cdots j_{n}}$ should all be different; otherwise, the AIF would be zero. Denote also

$$
\tilde{\mathcal{F}}_{\mathbf{j}}^{(n)}=\left(\tilde{\mathcal{B}}_{j_{n}} \mathcal{B}_{j_{n-1}} \cdots \mathcal{B}_{j_{1}}+\cdots+\mathcal{B}_{j_{n}} \cdots \mathcal{B}_{j_{2}} \tilde{\mathcal{B}}_{j_{1}}\right) \mathcal{F}
$$

Equations (4.1) and (4.2) lead then to

$$
\begin{aligned}
\partial_{t} \mathcal{F}_{\mathbf{j}}^{(n)}= & \tilde{\mathcal{F}}_{\mathbf{j}}^{(n)}-i\left(\tilde{\mathcal{C}}_{j_{n}} \mathcal{B}_{j_{n-1}} \cdots \mathcal{B}_{j_{1}}+\cdots\right. \\
& \left.+\mathcal{B}_{j_{n}} \cdots \mathcal{B}_{j_{2}} \tilde{\mathcal{C}}_{j_{1}}\right) \mathcal{F}-i \sum_{j}^{\prime} \mathcal{B}_{j_{n}} \cdots \mathcal{B}_{j_{1}} \mathcal{A}_{j} \mathcal{B}_{j} \mathcal{F} \\
= & \tilde{\mathcal{F}}_{\mathbf{j}}^{(n)}-i \sum_{k=1}^{n}(-)^{n-k} \tilde{\mathcal{C}}_{j_{k}} \mathcal{B}_{j_{n}} \cdots \mathcal{B}_{j_{k+1}} \\
& \times \mathcal{B}_{j_{k-1}} \cdots \mathcal{B}_{j_{1}} \mathcal{F}-i \sum_{j}^{\prime} \mathcal{A}_{j} \mathcal{B}_{j} \mathcal{B}_{j_{n}} \cdots \mathcal{B}_{j_{1}} \mathcal{F}
\end{aligned}
$$

The sum $\sum^{\prime}$ runs over all $j \neq j_{k} ; k=1, \cdots, n$. Denote

$$
\mathcal{F}_{\mathbf{j}_{k}}^{(n-1)} \equiv \mathcal{B}_{j_{n}} \cdots \mathcal{B}_{j_{k+1}} \mathcal{B}_{j_{k-1}} \cdots \mathcal{B}_{j_{1}} \mathcal{F}
$$

and

$$
\mathcal{F}_{\mathbf{j} j}^{(n+1)} \equiv \mathcal{B}_{j} \mathcal{B}_{j_{n}} \cdots \mathcal{B}_{j_{1}} \mathcal{F}
$$

Equation (4.10) can now be recast as

$$
\begin{aligned}
\partial_{t} \mathcal{F}_{\mathbf{j}}^{(n)}= & \tilde{\mathcal{F}}_{\mathbf{j}}^{(n)}-i \sum_{k=1}^{n}(-)^{n-k} \tilde{\mathcal{C}}_{j_{k}} \mathcal{F}_{\mathbf{j}_{k}}^{(n-1)} \\
& -i \sum_{j}^{\prime} \mathcal{A}_{\bar{j}} \mathcal{F}_{\mathbf{j} j}^{(n+1)}
\end{aligned}
$$

Apparently, an odd-tier AIF is a Grassmann variable, while an even-tier one an ordinary c-number. This is the Grassmann parity that will affect the explicit expressions of the $\mathcal{A}$ - and $\mathcal{C}$-terms in Eq. (4.12) in PI repression, and consequently their ADO counterparts in operator level; see Eqs. (4.15).

\section{B. Auxiliary density operators dynamics}

The $n^{\text {th }}$-order ADO $\rho_{\mathbf{j}}^{(n)}$ can now be defined via the AIF $\mathcal{F}_{\mathbf{j}}^{(n)}$ as [cf. Eq. (3.10)]

$$
\rho_{\mathbf{j}}^{(n)}(t) \equiv \mathcal{U}_{\mathbf{j}}^{(n)}\left(t, t_{0}\right) \rho\left(t_{0}\right)
$$

with

$$
\mathcal{U}_{\mathbf{j}}^{(n)}\left(\boldsymbol{\psi}, t ; \boldsymbol{\psi}_{0}, t_{0}\right) \equiv \int_{\boldsymbol{\psi}_{0}\left[t_{0}\right]}^{\boldsymbol{\psi}[t]} \mathcal{D} \boldsymbol{\psi} e^{i S[\psi]} \mathcal{F}_{\mathbf{j}}^{(n)}[\boldsymbol{\psi}] e^{-i S\left[\psi^{\prime}\right]}
$$

Similarly, the auxiliary operators $\tilde{\rho}_{\mathbf{j}}^{(n)}, \rho_{\mathbf{j}_{k}}^{(n-1)}$, and $\rho_{\mathbf{j} j}^{(n+1)}$ are defined via $\tilde{\mathcal{F}}_{\mathbf{j}}^{(n)}, \mathcal{F}_{\mathbf{j}_{k}}^{(n-1)}$, and $\mathcal{F}_{\mathbf{j} j}^{(n+1)}$, respectively. The leading term in the $n^{\text {th }}$-tier AIF such as $\mathcal{F}^{(n)}$ or $\tilde{\mathcal{F}}^{(n)}$ is of the $(2 n)^{\text {th }}$-order in the system-bath coupling; so is that in each individual $\rho_{\mathbf{j}}^{(n)}$ or $\tilde{\rho}_{\mathbf{j}}^{(n)}$.

The EOM for $\rho^{(n)}$ can then be readily obtained via Eq. (4.12) and (4.13). Together with Eq. (3.11) and setting $\rho^{(0)} \equiv \rho$, we have

$$
\begin{aligned}
& \dot{\rho}=-i \mathcal{L} \rho-i \sum_{j} \mathcal{A}_{\bar{j}} \rho_{j}^{(1)}, \\
& \dot{\rho}_{\mathbf{j}}^{(n)}=-i \mathcal{L} \rho_{\mathbf{j}}^{(n)}+\tilde{\rho}_{\mathbf{j}}^{(n)}-i \sum_{k=1}^{n}(-)^{n-k} \tilde{\mathcal{C}}_{j_{k}} \rho_{\mathbf{j}_{k}}^{(n-1)} \\
& -i \sum_{j}^{\prime} \mathcal{A}_{\bar{j}} \rho_{\mathbf{j} j}^{(n+1)} ; \quad n>0 .
\end{aligned}
$$

Here, $\mathcal{A}_{\bar{j}} \equiv \mathcal{A}_{\mu}^{\bar{\sigma}}$ and $\tilde{\mathcal{C}}_{j} \equiv \tilde{\mathcal{C}}_{\alpha \mu}^{\sigma}$ are superoperators. Their PI expressions that follow the Grassmann algebra are given in Eqs. (3.4) and (4.4), respectively. As the Grassmann parity associated with AIFs, the actions of these superoperators in Eq. (4.14) are given as

$$
\mathcal{A}_{\bar{j}} \rho^{(m)}=a_{\mu}^{\bar{\sigma}} \rho^{(m)}+(-)^{m} \rho^{(m)} a_{\mu}^{\bar{\sigma}}
$$

and

$$
\tilde{\mathcal{C}}_{j} \rho^{(m)}=\sum_{\nu}\left\{C_{\alpha \mu \nu}^{0, \sigma} a_{\nu}^{\sigma} \rho^{(m)}-(-)^{m} C_{\alpha \nu \mu}^{0, \bar{\sigma}} \rho^{(m)} a_{\nu}^{\sigma}\right\} .
$$

The $n$ indexes in $\rho_{\mathbf{j}}^{(n)} \equiv \rho_{j_{1} j_{2} \ldots j_{n}}^{(n)}$ should all be distinct, due to the Grassmann or Fermion anticommutation relation associated with the index swap. Recall that $j \equiv\{\alpha \mu \sigma\}$. As results, $\rho^{(n)}=0$, if $n>2 N_{\alpha} N_{c}$. Here, $N_{\alpha}$ denotes the number of electrodes; $N_{c}$ is the number of spin-orbitals that couple directly to the electrodes, [i.e, those of $\left.C_{\alpha \mu \mu}(t, \tau) \neq 0\right]$. The factor 2 arises from the two possible signs of $\sigma=+$ and - .

The EOM formalism presented in Eq. (4.14) is finite but yet to be closed due to the fact that $\left\{\tilde{\rho}_{\mathbf{j}}^{(n)}\right\}$, as defined via $\tilde{\mathcal{F}}_{\mathbf{j}}^{(n)}$ [Eq. (4.9)], involves both the $\mathcal{B}$ - and $\tilde{\mathcal{B}}$-types functionals. The desired hierarchy should be constructed only via the $\mathcal{B}$-type functionals. To obtain a closed HEOM formalism, it requires that $\tilde{\mathcal{B}}$-type functionals [Eq. (4.3)] be expressed in terms of the $\mathcal{B}$ 's [Eq. (3.5)]. This can be achieved via the extended Meier-Tannor parameterization method, $33,35,36,41,45$ in which correlation functions $C_{\alpha \mu \nu}^{\sigma}(t)$ are expanded in an exponential series. This method was adopted in our previous development of QDT formalism for bosonic bath cases, in which the path integral variables are all c-numbers ${ }^{35.36}$ We shall treat the parametrization approach to HEOM for Fermion reservoirs later; see Sec. VIII and Appendix C 


\section{The multiple-frequency dispersed hierarchy construction}

Proposed here is the MFD hierarchy scheme, in which

$$
\rho_{\mathbf{j}}^{(n)}(t)=\int d \omega_{1} \cdots \int d \omega_{n} \phi_{j_{1} \cdots j_{n}}^{(n)}\left(\omega_{1}, \cdots, \omega_{n} ; t\right) .
$$

The desired MFD-HEOM [cf. Eq. (5.1)] will be constructed for a proper

$$
\phi_{\mathbf{j}}^{(n)}(\boldsymbol{\omega} ; t) \equiv \phi_{j_{1} \cdots j_{n}}^{(n)}\left(\omega_{1}, \cdots, \omega_{n} ; t\right) .
$$

Let us start with the time-independent chemical potential case, in which the reservoir correlation functions is stationary, $C_{\alpha \mu \nu}^{\sigma}(t, \tau)=C_{\alpha \mu \nu}^{\sigma}(t-\tau)$, and can be expressed in terms of spectrum functions as Eq. (2.9). In this case, Eq. (3.6) can be recast as

$$
B_{j}(t ;\{\psi\})=\int d \omega \hat{B}_{j}(\omega, t ;\{\psi\}),
$$

with

$$
\hat{B}_{j}(\omega, t ;\{\psi\})=\sum_{\nu} \int_{t_{0}}^{t} d \tau e^{i \sigma \omega(t-\tau)} \Gamma_{\alpha \mu \nu}^{\sigma}(\omega) a_{\nu}^{\sigma}[\psi(\tau)] .
$$

The frequency-dispersed $\hat{B}_{j}^{\prime}$ is similar to $\hat{B}_{j}$, except that the $\Gamma_{\alpha \mu \nu}^{\sigma}$ oh the rhs of Eq. (4.19) is replaced by $\Gamma_{\alpha \mu \nu}^{\bar{\sigma} *}=\Gamma_{\alpha \nu \mu}^{\bar{\sigma}}$ [cf. the first identity of Eq. (2.10)]. As results, Eq. (3.5) can be expressed as

$$
\mathcal{B}_{j} \equiv \mathcal{B}_{\alpha \mu}^{\sigma}(t ;\{\boldsymbol{\psi}\})=\int d \omega \hat{\mathcal{B}}_{j}(\omega, t ;\{\boldsymbol{\psi}\})
$$

with $\hat{\mathcal{B}}_{j} \equiv-i\left(\hat{B}_{j}-\hat{B}_{j}^{\prime}\right)$.

In the presence of time-dependent chemical potentials applied on electrodes, the correlation functions include also the nonstationary phase factors [cf. Eq. (2.16) ]. The frequency dispersed $\hat{\mathcal{B}}_{j}$-functional reads now

$$
\begin{aligned}
\hat{\mathcal{B}}_{j}= & -i \sum_{\nu} \int_{t_{0}}^{t} d \tau e^{i \sigma \omega(t-\tau)} \exp \left[\sigma i \int_{\tau}^{t} d t^{\prime} \Delta_{\alpha}\left(t^{\prime}\right)\right] \\
& \times\left\{\Gamma_{\alpha \mu \nu}^{\sigma}(\omega) a_{\nu}^{\sigma}[\psi(\tau)]-\Gamma_{\alpha \nu \mu}^{\bar{\sigma}}(\omega) a_{\nu}^{\sigma}\left[\psi^{\prime}(\tau)\right]\right\} .
\end{aligned}
$$

It satisfies

$$
\partial_{t} \hat{\mathcal{B}}_{j}=i \sigma\left[\omega+\Delta_{\alpha}(t)\right] \hat{\mathcal{B}}_{j}+\mathcal{C}_{j}(\omega),
$$

with $\mathcal{C}_{j}(\omega)=\mathcal{C}_{j}(\omega ;\{\boldsymbol{\psi}[t]\})$ being

$$
\mathcal{C}_{j}=\sum_{\nu}\left\{\Gamma_{\alpha \mu \nu}^{\sigma}(\omega) a_{\nu}^{\sigma}[\psi(t)]-\Gamma_{\alpha \nu \mu}^{\bar{\sigma}}(\omega) a_{\nu}^{\sigma}\left[\psi^{\prime}(t)\right]\right\} .
$$

The $n^{\text {th }}$-tier AIFs, $\mathcal{F}_{\mathbf{j}}^{(n)}$ of Eq. (4.8), can now be dispersed as

$$
\mathcal{F}_{\mathbf{j}}^{(n)}=\int d \boldsymbol{\omega} \hat{\mathcal{F}}_{\mathbf{j}}^{(n)}(\boldsymbol{\omega} ; t ;\{\boldsymbol{\psi}\}),
$$

where [noting that $\left.\hat{\mathcal{B}}_{j_{k}} \equiv \hat{\mathcal{B}}_{j_{k}}\left(\omega_{k}, t ;\{\boldsymbol{\psi}\}\right)\right]$

$$
\hat{\mathcal{F}}_{\mathbf{j}}^{(n)}(\boldsymbol{\omega} ; t ;\{\boldsymbol{\psi}\}) \equiv \hat{\mathcal{B}}_{j_{n}} \cdots \hat{\mathcal{B}}_{j_{2}} \hat{\mathcal{B}}_{j_{1}} \mathcal{F}
$$

The proper MFD-ADO, $\phi_{\mathbf{j}}^{(n)}$ in Eq. (4.17), for closing the hierarchy is now determined as

$$
\phi_{\mathbf{j}}^{(n)}(\boldsymbol{\omega} ; t)=\hat{\mathcal{U}}_{\mathbf{j}}^{(n)}\left(\boldsymbol{\omega} ; t, t_{0}\right) \rho\left(t_{0}\right),
$$

with

$$
\hat{\mathcal{U}}_{\mathbf{j}}^{(n)}=\int_{\boldsymbol{\psi}_{0}\left[t_{0}\right]}^{\boldsymbol{\psi}[t]} \mathcal{D} \boldsymbol{\psi} e^{i S[\psi]} \hat{\mathcal{F}}_{\mathbf{j}}^{(n)} e^{-i S\left[\psi^{\prime}\right]} .
$$

Note that the Grassmann anticommutation relation is now manifested not only in the $j$-indexes, but also in the associated frequencies; cf. Eq. (4.25).

The time-derivative on Eq. (4.25) can be obtained as

$$
\begin{aligned}
\partial_{t} \hat{\mathcal{F}}_{\mathbf{j}}^{(n)}= & i \Omega_{\boldsymbol{\alpha} \boldsymbol{\sigma}}^{(n)} \hat{\mathcal{F}}_{\mathbf{j}}^{(n)}-i \sum_{k=1}^{n}(-)^{n-k} \mathcal{C}_{j_{k}}\left(\omega_{k}\right) \hat{\mathcal{F}}_{\mathbf{j}_{k}}^{(n-1)} \\
& -i \int d \omega \sum_{j} \mathcal{A}_{j} \hat{\mathcal{F}}_{\mathbf{j} j}^{(n+1)}(\boldsymbol{\omega}, \omega, t ;\{\boldsymbol{\psi}\}),
\end{aligned}
$$

with $\Omega_{\boldsymbol{\alpha} \boldsymbol{\sigma}}^{(n)}=\sum_{k} \sigma_{k}\left[\omega_{k}+\Delta_{\alpha_{k}}(t)\right]$. The MFD-HEOM for $\phi_{\mathbf{j}}^{(n)}$ can then be readily obtained.

\section{HIERARCHICAL EQUATIONS OF MOTION WITH MULTIPLE FREQUENCY DISPERSION}

The final MFD-HEOM formalism at operator level reads as (setting $\phi^{(-1)} \equiv \Omega^{(0)} \equiv 0$ and $\phi^{(0)} \equiv \rho$ )

$$
\begin{aligned}
\dot{\phi}_{\mathbf{j}}^{(n)}(\boldsymbol{\omega}, t)= & -i\left[\mathcal{L}-\Omega_{\boldsymbol{\alpha \sigma}}^{(n)}(\boldsymbol{\omega}, t)\right] \phi_{\mathbf{j}}^{(n)}(\boldsymbol{\omega}, t) \\
& -i \sum_{k=1}^{n}(-)^{n-k} \mathcal{C}_{j_{k}}\left(\omega_{k}\right) \phi_{\mathbf{j}_{k}}^{(n-1)}\left(\boldsymbol{\omega}_{k}^{\prime}, t\right) \\
& -i \int d \omega \sum_{j \equiv\{\alpha \mu \sigma\}} \mathcal{A}_{\mu}^{\bar{\sigma}} \phi_{\mathbf{j} j}^{(n+1)}(\boldsymbol{\omega}, \omega, t) .
\end{aligned}
$$

Here, $\boldsymbol{\omega}_{k}^{\prime} \equiv\left\{\omega_{1} ; \cdots, \omega_{k-1}, \omega_{k+1} ; \cdot ; \omega_{n}\right\}$,

$$
\Omega_{\boldsymbol{\alpha} \boldsymbol{\sigma}}^{(n)}(\boldsymbol{\omega}, t)=\sum_{k=1}^{n} \sigma_{k}\left[\omega_{k}+\Delta_{\alpha_{k}}(t)\right],
$$

and [cf. Eqs. (4.15) and (4.23)]

$$
\begin{aligned}
& \mathcal{A}_{\mu}^{\bar{\sigma}} \phi^{(m)}=a_{\mu}^{\bar{\sigma}} \phi^{(m)}+(-)^{m} \phi^{(m)} a_{\mu}^{\bar{\sigma}}, \\
& \mathcal{C}_{j}(\omega) \phi^{(m)}=\sum_{\nu}\left[\Gamma_{\alpha \mu \nu}^{\sigma}(\omega) a_{\nu}^{\sigma} \phi^{(m)}\right. \\
& \left.-(-)^{m} \Gamma_{\alpha \nu \mu}^{\bar{\sigma}}(\omega) \phi^{(m)} a_{\nu}^{\sigma}\right] .
\end{aligned}
$$


The initial conditions to Eq. (5.1) are in fact those of steady-state solutions; see Sec. IX] for details. The transient current [Eq. (3.13)] is evaluated via the first-tier frequency-dispersed $\mathrm{ADO}, \phi_{j}^{(1)}(\omega, t) \equiv \phi_{\alpha \mu}^{\sigma}(\omega, t)$, as

$$
I_{\alpha}(t)=-2 \operatorname{Im} \int d \omega \sum_{\mu} \operatorname{tr}_{\mathrm{s}}\left\{a_{\mu} \phi_{\alpha \mu}^{+}(\omega, t)\right\} .
$$

As inferred from Eq. (4.21) and the first identity of Eq. (2.10), we have (denoting $\bar{j} \equiv\{\alpha \mu \bar{\sigma}\}$ )

$$
\left[\phi_{j_{1} \cdots j_{n}}^{(n)}\right]^{\dagger}=\phi_{\bar{j}_{n} \cdots \bar{j}_{1}}^{(n)}=(-)^{\left[\frac{n}{2}\right]} \phi_{\bar{j}_{1} \cdots \bar{j}_{n}}^{(n)},
$$

which can be abbreviated as $\left[\phi_{\mathbf{j}}^{(n)}\right]^{\dagger}=(-)^{\left[\frac{n}{2}\right]} \phi_{\overline{\mathbf{j}}}^{(n)}$, with $\left[\frac{n}{2}\right] \equiv \operatorname{Int}(n / 2)$ being for the number of index-swaps involved. This is the generalization of Eq. (3.12). The Grassmann anticommutation relation in $\phi_{\mathbf{j}}^{(n)}(\boldsymbol{\omega} ; t)$ involves not only the $j$-indexes, but also the associated frequencies, such as $\phi_{j^{\prime} j}\left(\omega^{\prime}, \omega ; t\right)=-\phi_{j j^{\prime}}\left(\omega, \omega^{\prime} ; t\right)$. As results, $\phi_{j j} \neq 0$, although its double-frequency integral vanishes.

The MFD-HEOM formalism is exact, but with an infinite hierarchy. The truncation is however rather trivial, 35 as the leading term in an $n^{\text {th }}$-tier $\operatorname{ADO} \phi^{(n)}$ is of the $(2 n)^{\text {th }}$-order system-reservoir coupling [cf. Eq. (4.25)]. In practice, one can set all $\phi^{\left(n>N_{\text {trun }}\right)} \approx 0$, followed by the convergence test with increasing $N_{\text {trun }}$ for the anchor tier of the hierarchy. We will show in Sec.VII that the present theory with $N_{\text {trun }}=2$ recovers the realtime diagrammatic formalism developed by Schön and coworkers $8,9,10,11,12$ Also, we will see soon that in the absence of Coulomb interaction, the Grassmann parity of $\mathcal{A}_{\mu}^{\bar{\sigma}}$ defined in Eq. (5.3a) leads to $\phi^{(3)}=0$ without approximation; the present theory is exact of $n_{\max }=2$ for single-particle systems.

\section{EQUATIONS OF MOTION FOR SINGLE-PARTICLE HAMILTONIAN SYSTEMS}

\section{A. Auxiliary single-particle density matrices}

Consider a single-particle system, described by

$$
H=\sum_{\mu \nu} h_{\mu \nu} a_{\mu}^{\dagger} a_{\nu}
$$

The electronic dynamics in such a system can be characterized by reduced single-particle density matrix (RSPDM), $\varrho$, defined via its elements of

$$
\varrho_{\mu \nu}(t) \equiv \operatorname{tr}_{\mathrm{s}}\left[a_{\nu}^{\dagger} a_{\mu} \rho(t)\right]
$$

Presented in Appendix $\mathrm{B}$ is the derivation of RSPDM dynamics via MFD-HEOM, which is of $n_{\max }=2$ for the single-particle system without approximation. This results from the Grassmann parity associated $\mathcal{A}_{\mu}^{\bar{\sigma}}$ [Eq. (5.3a)], whose action on single-particle operators via
Eq. (5.1) will terminate at $n_{\max }=2$. Only the first- and the second-tier auxiliary density matrices, $\varphi_{\alpha}$ and $\varphi_{\alpha^{\prime} \alpha}$, are required for a single-particle system. These auxiliary frequency-resolved density matrices can be defined via their elements of

$$
\left[\varphi_{\alpha}(\omega, t)\right]_{\mu \nu} \equiv \operatorname{tr}_{\mathrm{S}}\left[a_{\mu} \phi_{\alpha \nu}^{+}(\omega, t)\right]
$$

and

$$
\left[\varphi_{\alpha^{\prime} \alpha}\left(\omega^{\prime}, \omega, t\right)\right]_{\mu \nu} \equiv \operatorname{tr}_{\mathrm{s}}\left[\phi_{\alpha \nu, \alpha^{\prime} \mu}^{+,-}\left(\omega, \omega^{\prime}, t\right)\right]
$$

It can be shown via Eq.

$$
\left[\boldsymbol{\varphi}_{\alpha^{\prime} \alpha}\left(\omega^{\prime}, \omega, t\right)\right]^{\dagger}=\boldsymbol{\varphi}_{\alpha \alpha^{\prime}}\left(\omega, \omega^{\prime}, t\right) .
$$

The final results are summarized as follows.

$$
\begin{aligned}
i \dot{\boldsymbol{\varrho}}= & {[\boldsymbol{h}, \boldsymbol{\varrho}]-\sum_{\alpha} \int d \omega\left[\boldsymbol{\varphi}_{\alpha}(\omega, t)-\boldsymbol{\varphi}_{\alpha}^{\dagger}(\omega, t)\right], } \\
i \dot{\boldsymbol{\varphi}}_{\alpha}= & {\left[\boldsymbol{h}-\omega-\Delta_{\alpha}(t)\right] \boldsymbol{\varphi}_{\alpha}+\boldsymbol{S}_{\alpha}(\omega)-\boldsymbol{\varrho} \boldsymbol{J}_{\alpha}(\omega) } \\
& +\sum_{\alpha^{\prime}} \int d \omega^{\prime} \boldsymbol{\varphi}_{\alpha^{\prime} \alpha}\left(\omega^{\prime}, \omega, t\right), \\
i \dot{\boldsymbol{\varphi}}_{\alpha^{\prime} \alpha}= & -\left[\omega+\Delta_{\alpha}(t)-\omega^{\prime}-\Delta_{\alpha^{\prime}}(t)\right] \boldsymbol{\varphi}_{\alpha^{\prime} \alpha} \\
& +\boldsymbol{J}_{\alpha^{\prime}}\left(\omega^{\prime}\right) \boldsymbol{\varphi}_{\alpha}(\omega, t)-\boldsymbol{\varphi}_{\alpha^{\prime}}^{\dagger}\left(\omega^{\prime}, t\right) \boldsymbol{J}_{\alpha}(\omega) .
\end{aligned}
$$

Here,

$$
\boldsymbol{S}_{\alpha}(\omega) \equiv f_{\alpha}(\omega) \boldsymbol{J}_{\alpha}(\omega)=\left(\boldsymbol{\Gamma}_{\alpha}^{+}\right)^{T}
$$

with $f_{\alpha}(\omega)$ being the Fermi distribution function, and $\boldsymbol{J}_{\alpha}(\omega)=\boldsymbol{J}_{\alpha}^{\dagger}(\omega)$ [Eq. (2.11)] the spectral density function matrix of the reservoir $\alpha$. The last identity in Eq. (6.7) states that $\boldsymbol{S}_{\alpha}$ is equivalent to the $\boldsymbol{\Gamma}_{\alpha}^{+}$-matrix transpose. Note that Eq. (6.6) can be recast in the standard form of linearly coupled equations, see Eq. (B10).

The transient current of Eq. 3.13) from the $\alpha$-lead to the system can be expressed as

$$
I_{\alpha}(t)=-2 \operatorname{Im} \int d \omega \operatorname{tr}\left[\boldsymbol{\varphi}_{\alpha}(\omega, t)\right] .
$$

The matrix trace is used here. Together with Eq. 6.6b), the above expression leads to $\operatorname{tr} \dot{\boldsymbol{\varrho}}(t)=\sum_{\alpha} I_{\alpha}(t)$. This is the flux conservation relation.

\section{B. Steady-state current and the Landauer-Büttiker formula}

Consider now the stationary solutions, $\varrho=\varrho(t \rightarrow \infty)$ and $\varphi(\omega) \equiv \varphi(\omega, t \rightarrow \infty)$, to Eq. (6.6) in the absence of time-dependent external bias potentials. The steadystate solution to Eq. (6.6c) is

$$
\boldsymbol{\varphi}_{\alpha^{\prime} \alpha}\left(\omega^{\prime}, \omega\right)=\frac{\boldsymbol{J}_{\alpha^{\prime}}\left(\omega^{\prime}\right) \boldsymbol{\varphi}_{\alpha}(\omega)-\boldsymbol{\varphi}_{\alpha^{\prime}}^{\dagger}\left(\omega^{\prime}\right) \boldsymbol{J}_{\alpha}(\omega)}{\omega-\omega^{\prime}+i 0^{+}}
$$


To proceed, let $x(\omega)=\sum_{\alpha} x_{\alpha}(\omega)$; where $x \in$ $\{\boldsymbol{J}, \boldsymbol{S}, \boldsymbol{\varphi}\}$. Adopt also the common definitions of retarded self-energy $\boldsymbol{\Sigma}$ and Green's function $\boldsymbol{G}$ as

$$
\boldsymbol{\Sigma}(\omega) \equiv \int d \omega^{\prime} \frac{\boldsymbol{J}\left(\omega^{\prime}\right)}{\omega-\omega^{\prime}+i 0^{+}},
$$

and

$$
\boldsymbol{G}(\omega) \equiv[\omega-\boldsymbol{h}-\boldsymbol{\Sigma}(\omega)]^{-1} .
$$

Substituting Eqs. (6.9) and (6.10) into Eq. (6.6b) in steady state leads to

$$
\begin{aligned}
\boldsymbol{G}^{-1}(\omega) \boldsymbol{\varphi}_{\alpha}(\omega)= & \boldsymbol{S}_{\alpha}(\omega)-\varrho \boldsymbol{J}_{\alpha}(\omega) \\
& -\int d \omega^{\prime} \frac{\boldsymbol{\varphi}^{\dagger}\left(\omega^{\prime}\right)}{\omega-\omega^{\prime}+i 0^{+}} J_{\alpha}(\omega) .
\end{aligned}
$$

Summing over $\alpha$ in the above equation, followed by some simple algebra, will obtain the relation of

$$
\int d \omega^{\prime} \frac{\boldsymbol{\varphi}^{\dagger}\left(\omega^{\prime}\right)}{\omega-\omega^{\prime}+i 0^{+}}=\boldsymbol{S} \boldsymbol{J}^{-1}-\varrho-\boldsymbol{G}^{-1} \boldsymbol{\varphi} \boldsymbol{J}^{-1} .
$$

Therefore

$$
\boldsymbol{\varphi}_{\alpha}=\boldsymbol{G}\left(\boldsymbol{S}_{\alpha}-\boldsymbol{S} \boldsymbol{J}^{-1} \boldsymbol{J}_{\alpha}\right)+\boldsymbol{\varphi} \boldsymbol{J}^{-1} \boldsymbol{J}_{\alpha} .
$$

For electrodes of similarity, $\boldsymbol{J}_{\alpha}(\omega) \sim \boldsymbol{J}_{\alpha^{\prime}}(\omega)$, up to trivial constants, the last term in Eq. (6.13) does not contribute to current of Eq. (6.8), as it satisfies

$$
\operatorname{Im} \int d \omega \operatorname{tr}\left(\boldsymbol{\varphi} \boldsymbol{J}^{-1} \boldsymbol{J}_{\alpha}\right) \propto \operatorname{Im} \int d \omega \operatorname{tr} \boldsymbol{\varphi}=0 .
$$

Thus, the steady-state current assumes

$$
I_{\alpha}=-2 \operatorname{Im} \int d \omega \operatorname{tr}\left[\boldsymbol{G}\left(\boldsymbol{S}_{\alpha}-\boldsymbol{S J}^{-1} \boldsymbol{J}_{\alpha}\right)\right] .
$$

Consider now the current contribution from lead $\mathrm{L}$ in a two-terminal setup. The quantity in the parentheses in the rhs of Eq. (6.13) can be evaluated as

$$
\boldsymbol{S}_{\mathrm{L}}-\boldsymbol{S J}^{-1} \boldsymbol{J}_{\mathrm{L}}=\left[f_{\mathrm{L}}(\omega)-f_{\mathrm{R}}(\omega)\right] \overline{\boldsymbol{J}}(\omega),
$$

with

$$
[\overline{\boldsymbol{J}}(\omega)]^{-1}=\left[\boldsymbol{J}_{\mathrm{L}}(\omega)\right]^{-1}+\left[\boldsymbol{J}_{\mathrm{R}}(\omega)\right]^{-1} .
$$

The identity $\boldsymbol{A}(\boldsymbol{A}+\boldsymbol{B})^{-1} \boldsymbol{B}=\left(\boldsymbol{A}^{-1}+\boldsymbol{B}^{-1}\right)^{-1}$ is used here. Thus Eq. (6.14) for L-lead reads

$$
I_{\mathrm{L}}=-2 \operatorname{Im} \int d \omega \operatorname{tr}\left[\left(f_{\mathrm{L}}-f_{\mathrm{R}}\right) \boldsymbol{G} \overline{\boldsymbol{J}}\right] .
$$

This is exactly the Landauer-Büttiker's result, 1,46,47,48 with the original factor of $(2 \pi)^{-1}$ being included now in the reservoir spectral density matrix.

\section{RELATION TO THE REAL-TIME DIAGRAMMATIC FORMALISM}

In this section we shall show that the real-time diagrammatic formalism developed by Schön and coworkers $8,9,10,11,12$ corresponds to a second-tier approximation, i.e., setting all $\phi^{(n \geq 3)}=0$, within the framework of the present MFD-HEOM of Eq. (5.1). For clarity, we choose for demonstration the same system as Ref.11, where Kondo physics was studied via steady-state twoterminal current measurement in the strong Coulomb interaction regime. The Anderson impurity Hamiltonian is adopted for the quantum dot system,

$$
H=\sum_{\mu} \epsilon_{\mu} \hat{n}_{\mu}+U \sum_{\mu<\mu^{\prime}} \hat{n}_{\mu} \hat{n}_{\mu^{\prime}}
$$

Here, $\hat{n}_{\mu}=a_{\mu}^{\dagger} a_{\mu}$, with $\mu$ being the spin index; thus $C_{\alpha \mu \nu}^{\sigma}(t)=0$ if $\mu \neq \nu$, and $\Gamma_{\alpha \mu \nu}^{\sigma}(\omega)=\Gamma_{\alpha \mu}^{\sigma}(\omega) \delta_{\mu \nu}$. In the strong Coulomb interaction regime, $a_{\mu}=|0\rangle\langle\mu|$, as the basis set involves only the zero and single occupation dot states and can be denoted as $\{|s\rangle ; s=0, \mu\}$. As results, $\rho \equiv \phi^{(0)}$ and its associated ADOs $\phi_{\mathbf{j}}^{(n>0)}$ in Eq. (5.1) are all $(m+1) \times(m+1)$ matrices, with $m$ being the number of spin states. The measured current $I=I_{\mathrm{L}}-I_{\mathrm{R}}$ is then of [cf. Eq. (5.4)]

$$
I_{\alpha}=-2 \operatorname{Im} \int d \omega \sum_{\mu}\left[\phi_{\alpha \mu}^{+}(\omega)\right]_{\mu 0},
$$

with $\left[\phi_{\alpha \mu}^{\sigma}(\omega)\right]_{s s^{\prime}} \equiv\left\langle s\left|\phi_{\alpha \mu}^{\sigma}(\omega)\right| s^{\prime}\right\rangle$ being the matrix element of the first-tier ADO. Note also that the reduced density matrix in a steady-state is diagonal, $\rho_{s s^{\prime}}^{\text {st }}=$ $\delta_{s s^{\prime}} \rho_{s s}^{\text {st }} \equiv \delta_{s s^{\prime}} P_{s}$, with $\sum_{s} P_{s}=P_{0}+\sum_{\mu} P_{\mu}=1$, as the density matrix in the dot-state representation is normalized. Under the steady-state condition, Eq. (5.1) with $n=0$ reads

$$
0=\dot{P}_{\mu}=-2 \operatorname{Im} \sum_{\alpha} \int d \omega\left[\phi_{\alpha \mu}^{+}(\omega)\right]_{\mu 0},
$$

while it for the first-tier auxiliary density matrix element, $\left\langle\mu\left|\dot{\phi}_{\alpha \mu}^{+}(\omega)\right| 0\right\rangle=0$, reads

$$
\begin{aligned}
0= & \left(\epsilon_{\mu}-\omega-i 0^{+}\right)\left[\phi_{\alpha \mu}^{+}(\omega)\right]_{\mu 0}+\Gamma_{\alpha \mu}^{+}(\omega) P_{0}-\Gamma_{\alpha \mu}^{-}(\omega) P_{\mu} \\
& +\int d \omega^{\prime}\left\{\left[\phi_{\alpha \mu \mu}^{+-}\left(\omega, \omega^{\prime}\right)\right]_{00}+\sum_{\mu^{\prime}}\left[\phi_{\alpha \mu \mu^{\prime}}^{+-}\left(\omega, \omega^{\prime}\right)\right]_{\mu \mu^{\prime}}\right\} .
\end{aligned}
$$

Here, $\phi_{\alpha \mu \mu^{\prime}}^{+-} \equiv \sum_{\alpha^{\prime}} \phi_{\alpha \mu, \alpha^{\prime} \mu^{\prime}}^{+,-}$are the coupling second-tier ADOs. The above equation does not involve $\phi_{\alpha \mu \alpha^{\prime} \mu^{\prime}}^{++}$, as $\left\langle\mu\left|\left[a_{\mu^{\prime}}, \phi_{\alpha \mu \alpha^{\prime} \mu^{\prime}}^{++}\left(\omega, \omega^{\prime}\right)\right]\right| 0\right\rangle=0$. Denote in the following are also $\Gamma_{\mu}^{ \pm} \equiv \sum_{\alpha} \Gamma_{\alpha \mu}^{ \pm}$and $\phi_{\mu}^{ \pm} \equiv \sum_{\alpha} \phi_{\alpha \mu}^{ \pm}$.

Presented in the following are the analytical results under the second-tier approximation, in which we set the third-tier ADOs $\phi^{(3)}=0$. Thus, the second-tier ADOs 
are determined completely by the first-tier ADOs. In the steady-state condition, we obtain

$$
\begin{aligned}
{\left[\phi_{\alpha \mu \mu}^{+-}\right]_{00} } & \approx \frac{\Gamma_{\mu}^{-}\left(\omega^{\prime}\right)\left[\phi_{\alpha \mu}^{+}(\omega)\right]_{\mu 0}-\Gamma_{\alpha \mu}^{-}(\omega)\left[\phi_{\mu}^{+}\left(\omega^{\prime}\right)\right]_{\mu 0}^{*}}{\omega-\omega^{\prime}+i 0^{+}}, \\
{\left[\phi_{\alpha \mu \mu^{\prime}}^{+-}\right]_{\mu \mu^{\prime}} } & \approx \frac{\Gamma_{\mu^{\prime}}^{+}\left(\omega^{\prime}\right)\left[\phi_{\alpha \mu}^{+}(\omega)\right]_{\mu 0}-\Gamma_{\alpha \mu}^{+}(\omega)\left[\phi_{\mu^{\prime}}^{+}\left(\omega^{\prime}\right)\right]_{\mu^{\prime} 0}^{*}}{\omega-\omega^{\prime}-\epsilon_{\mu}+\epsilon_{\mu^{\prime}}+i 0^{+}} .
\end{aligned}
$$

Substituted into Eq. (7.4) leads to

$$
\begin{aligned}
{\left[\phi_{\alpha \mu}^{+}(\omega)\right]_{\mu 0}=} & \Pi_{\mu}(\omega)\left[\Gamma_{\alpha \mu}^{+}(\omega) P_{0}-\Gamma_{\alpha \mu}^{-}(\omega) P_{\mu}\right. \\
- & \Gamma_{\alpha \mu}^{-}(\omega) \int d \omega^{\prime} \frac{\left[\phi_{\mu}^{+}\left(\omega^{\prime}\right)\right]_{\mu 0}^{*}}{\omega-\omega^{\prime}+i 0^{+}}-\Gamma_{\alpha \mu}^{+}(\omega) \\
& \left.\times \sum_{\mu^{\prime}} \int d \omega^{\prime} \frac{\left[\phi_{\mu^{\prime}}^{+}\left(\omega^{\prime}\right)\right]_{\mu^{\prime} 0}^{*}}{\omega-\omega^{\prime}-\omega_{\mu \mu^{\prime}}+i 0^{+}}\right]
\end{aligned}
$$

Here $\omega_{\mu \mu^{\prime}} \equiv \epsilon_{\mu}-\epsilon_{\mu^{\prime}}$, and

$$
\Pi_{\mu}(\omega)=\frac{1}{\omega-\epsilon_{\mu}-\Sigma_{\mu}(\omega)+i 0^{+}},
$$

with

$\Sigma_{\mu}(\omega) \equiv \int d \omega^{\prime}\left[\frac{\Gamma_{\mu}^{-}\left(\omega^{\prime}\right)}{\omega-\omega^{\prime}+i 0^{+}}+\sum_{\mu^{\prime}} \frac{\Gamma_{\mu^{\prime}}^{+}\left(\omega^{\prime}\right)}{\omega-\omega^{\prime}-\omega_{\mu \mu^{\prime}}+i 0^{+}}\right]$.

To make connections to the real-time diagrammatic formulation presented in Ref.11, we denote

$$
\left[\phi_{\alpha \mu}^{+}(\omega)\right]_{\mu 0} \equiv \sum_{s} Y_{\mu 0}^{s}(\alpha, \mu, \omega) P_{s}
$$

Here $Y_{\mu 0}^{s}(\alpha, \mu, \omega)$ is identical to the $\phi_{s, 0}^{s, \mu}(\alpha, \mu, \omega)$ in Ref.11. which is associated with the tunneling of an electron of spin $\mu$ and energy $\omega$, from $\alpha$-reservoir to the dot, provided the initial state of $\rho_{s s}=P_{s}$. Inserting Eq. (7.8) to Eq. (7.5) leads to

$$
\begin{aligned}
Y_{\mu 0}^{s}=\Pi_{\mu}(\omega)\left[\Gamma_{\alpha \mu}^{+}(\omega) \delta_{s 0}-\Gamma_{\alpha \mu}^{-}(\omega) \delta_{s \mu}\right. \\
-\Gamma_{\alpha \mu}^{-}(\omega) \int d \omega^{\prime} \frac{\left[Y_{\mu 0}^{s}\left(\mu, \omega^{\prime}\right)\right]^{*}}{\omega-\omega^{\prime}+i 0^{+}} \\
\left.-\Gamma_{\alpha \mu}^{+}(\omega) \sum_{\mu^{\prime}} \int d \omega^{\prime} \frac{\left[Y_{\mu^{\prime} 0}^{s}\left(\mu^{\prime}, \omega^{\prime}\right)\right]^{*}}{\omega-\omega^{\prime}-\omega_{\mu \mu^{\prime}}+i 0^{+}}\right]
\end{aligned}
$$

with $Y_{\mu 0}^{s}(\mu, \omega) \equiv \sum_{\alpha^{\prime}} Y_{\mu 0}^{s}\left(\alpha^{\prime}, \mu, \omega\right)$ adopted in the rhs. Equation (7.9) is identical to the key results, Eqs. (50)-(51) in Ref.11. We have thus demonstrated that the second-tier approximation of the present MFDHEOM [Eq. (5.1)] does recover the real-time diagrammatic formalism $\underline{8,9,10,11,12}$

\section{HIERARCHICAL EQUATIONS OF MOTION WITH PARAMETRIZATION}

\section{A. Non-Markovian reservoirs via parameterization}

In this section, we consider an alternative hierarchy construction based on exponential series expansion of reservoir correlation functions. The resulting HEOM formalism will completely be in time domain, thus has the advantage in numerical implementation. The exponential series expansion of reservoirs can be achieved via the extended Meier-Tannor parametrization method, $, 33,41,45$ which has been used in the construction of HEOM for systems coupled with bosonic bath 35,36

The fermionic reservoir parametrization starts with the following form of spectral density functions,

$$
J_{\alpha \mu \nu}^{\sigma}(\omega)=\sum_{k=0}^{K} \frac{\left(\Gamma_{\alpha k}^{\mu \nu}+\sigma i \bar{\Gamma}_{\alpha k}^{\mu \nu}\right)\left(W_{\alpha k}^{\mu \nu}\right)^{2}}{\left(\omega+\sigma \Omega_{\alpha k}^{\mu \nu}\right)^{2}+\left(W_{\alpha k}^{\mu \nu}\right)^{2}} .
$$

Involving parameters are all real and positive (except for the Drude term with $k=0$ and $\Omega_{\alpha 0}^{\mu \nu} \equiv 0$ ), and due to $J_{\alpha \mu \nu}^{\sigma}=J_{\alpha \nu \mu}^{\sigma *}$, they satisfy

$$
\left(\Gamma_{\alpha k}^{\mu \nu}, W_{\alpha k}^{\mu \nu}, \Omega_{\alpha k}^{\mu \nu}, \bar{\Gamma}_{\alpha k}^{\mu \nu}\right)=\left(\Gamma_{\alpha k}^{\nu \mu}, W_{\alpha k}^{\nu \mu}, \Omega_{\alpha k}^{\nu \mu},-\bar{\Gamma}_{\alpha k}^{\nu \mu}\right) .
$$

The corresponding stationary components of correlation functions can then be obtained via the FDT [Eq. (2.15)] using the contour integration method. We have

$$
C_{\alpha \mu \nu}^{\sigma}(t)=\sum_{k=0}^{K} \eta_{\alpha \mu \nu k}^{\sigma} e^{-\gamma_{\alpha \mu \nu k}^{\sigma} t}+\sum_{m=1}^{M} \check{\eta}_{\alpha \mu \nu m}^{\sigma} e^{-\check{\gamma}_{\alpha m}^{\sigma} t} .
$$

The first term arises from the poles of the spectral density functions, with ${ }^{36}$

$$
\begin{aligned}
\gamma_{\alpha \mu \nu k}^{\sigma} & =W_{\alpha k}^{\mu \nu}-\sigma i \Omega_{\alpha k}^{\mu \nu}, \\
\eta_{\alpha \mu \nu k}^{\sigma} & =\frac{\left(\Gamma_{\alpha k}^{\mu \nu}+\sigma i \bar{\Gamma}_{\alpha k}^{\mu \nu}\right) W_{\alpha k}^{\mu \nu}}{1+\exp \left[i \beta_{\alpha}\left(\gamma_{\alpha \mu \nu k}^{\sigma}+\sigma i \mu_{\alpha}\right)\right]} .
\end{aligned}
$$

Note that $\eta_{\alpha \mu \nu k}^{\bar{\sigma} *}=e^{i \beta_{\alpha}\left(\gamma_{\alpha \mu \nu}^{\sigma}+\sigma i \mu_{\alpha}\right)} \eta_{\alpha \mu \nu k}^{\sigma}$. The second term in Eq. (8.2), with $M \rightarrow \infty$ in principle, arises from the Matsubara poles. The involving parameters are ${ }^{36}$

$$
\begin{aligned}
\check{\gamma}_{\alpha m}^{\sigma} & =\beta_{\alpha}^{-1}(2 m-1) \pi-\sigma i \mu_{\alpha}, \\
\check{\eta}_{\alpha \mu \nu m}^{\sigma} & =\frac{2}{i \beta_{\alpha}} J_{\alpha \mu \nu}^{\sigma}\left(-i \check{\gamma}_{\alpha m}^{\sigma}\right)=-\check{\eta}_{\alpha \mu \nu m}^{\bar{\sigma} *} .
\end{aligned}
$$

The last identity arises from the symmetry relation of Fermion spectral density functions.

The dissipation functional [Eq. (3.3b)] is now decomposed according to Eq. (8.2) as

$$
\mathcal{R}=i \sum_{\mathbf{k}} \mathcal{A}_{\mu}^{\bar{\sigma}} \mathcal{B}_{\mathbf{k}}+i \sum_{\mathbf{m}} \mathcal{A}_{\mu}^{\bar{\sigma}} \check{\mathcal{B}}_{\mathbf{m}} .
$$

with $\mathbf{k} \equiv(\alpha \mu \nu \sigma k)$ and $\mathbf{m} \equiv(\alpha \mu \sigma m)$; see Appendix $\mathbf{C}$ for details. Presented there is also the derivation of HEOM formalism to be summarized as follows. 


\section{B. HEOM via parametrization: Final results}

The final HEOM formalism via parametrization of Eq. (8.1) can be written in the following compact form:

$$
\dot{\rho}_{\mathrm{n}}=-\left[i \mathcal{L}+\gamma_{\mathrm{n}}(t)\right] \rho_{\mathrm{n}}+\rho_{\mathrm{n}}^{\{-\}}+\rho_{\mathrm{n}}^{\{+\}},
$$

with $\gamma_{0}=\rho_{-1} \equiv 0$, and $\rho_{0} \equiv \rho$ the primary reduced density operator. The subscript $\mathbf{n} \equiv\left(\mathbf{k}_{1} \cdots \mathbf{k}_{p}, \mathbf{m}_{1} \cdots \mathbf{m}_{q}\right)$ denotes an ordered set of indexes, with

$$
\mathbf{k}=(\alpha \mu \nu \sigma k) \text { and } \mathbf{m}=(\alpha \mu \sigma m),
$$

arising from the two distinct components of the reservoir correlation functions [cf. Eq. (8.2) or (8.5)]. Note that

$$
\rho_{\mathrm{n}} \equiv \rho_{\mathbf{k}_{1} \cdots \mathbf{k}_{p}, \mathbf{m}_{1} \cdots \mathbf{m}_{q}}^{(n)} ; \quad p+q=n,
$$

is an $n^{\text {th }}$-tier ADO; see Eq. C7 for its associating AIF. It satisfies the Hermitian conjugate relation of [cf. Eq. (5.5) ]

$$
\left[\rho_{\mathbf{k}_{1} \cdots \mathbf{k}_{p}, \mathbf{m}_{1} \cdots \mathbf{m}_{q}}^{(n)}\right]^{\dagger}=(-)^{\left[\frac{n}{2}\right]} \rho_{\overline{\mathbf{k}}_{1} \cdots \overline{\mathbf{k}}_{p}, \overline{\mathbf{m}}_{1} \cdots \overline{\mathbf{m}}_{q}}^{(n)},
$$

where $\overline{\mathbf{k}}=(\alpha \mu \nu \bar{\sigma} k)$ and $\overline{\mathbf{m}}=(\alpha \mu \bar{\sigma} m)$.

The parameter $\gamma_{\mathrm{n}}$ in Eq. (8.6) collects the complex "damping" parameters of the involving reservoir correlation functions, see Eqs. (C2) and (C5). It reads

$$
\begin{aligned}
\gamma_{\mathbf{n}}(t)= & \sum_{j=1}^{p}\left[\gamma_{\alpha \mu \nu k}^{\sigma}-\sigma i \Delta_{\alpha}(t)\right]_{j ;\{\alpha, \mu, \nu, \sigma, k \in \mathbf{k}\}} \\
& +\sum_{l=1}^{q}\left[\check{\gamma}_{\alpha m}^{\sigma}-\sigma i \Delta_{\alpha}(t)\right]_{l ;\{\alpha, \sigma, m \in \mathbf{m}\}} .
\end{aligned}
$$

It contains not only the stationary components as Eq. (8.2), but also the nonstationary contributions. The latter are described by the time-dependent chemical potentials $\Delta_{\alpha}(t)$, applied on top of the constant $\mu_{\alpha}$ on electrodes; see Eq. (2.16).

The tier-down term in Eq. (8.6) reads

$$
\rho_{\mathrm{n}}^{\{-\}}=-i \sum_{j=1}^{p}(-)^{n-j} \mathcal{C}_{\mathbf{k}_{j}} \rho_{\mathrm{n}_{j}^{-}}-i \sum_{l=1}^{q}(-)^{q-l} \check{\mathcal{C}}_{\mathbf{m}_{l}} \rho_{\check{\mathbf{n}}_{l}^{-}},
$$

with the $(n-1)^{\text {th }}$-tier ADOs of

$$
\begin{aligned}
\rho_{\mathbf{n}_{j}^{-}} & \equiv \rho_{\mathbf{k}_{1} \cdots \mathbf{k}_{j-1}}^{(n-1)} \mathbf{k}_{j+1} \cdots \mathbf{k}_{p}, \mathbf{m}_{1} \cdots \mathbf{m}_{q} \\
\rho_{\check{\mathbf{n}}_{l}^{-}} & \equiv \rho_{\mathbf{k}_{1} \cdots \mathbf{k}_{p}, \mathbf{m}_{1} \cdots \mathbf{m}_{l-1}}^{(n-1)} \mathbf{m}_{l+1} \cdots \mathbf{m}_{q}
\end{aligned}
$$

$\mathcal{C}_{\mathbf{k}_{j}}$ and $\check{\mathcal{C}}_{\mathbf{m}_{l}}$ in Eq. (8.10) are the Liouville-space operator counterparts of Eq. (C6) in PI representation, with the Grassmann parity associated actions of

$$
\begin{aligned}
\mathcal{C}_{\mathbf{k}} \rho_{\mathrm{n}^{-}} & \equiv \eta_{\alpha \mu \nu k}^{\sigma} a_{\nu}^{\sigma} \rho_{\mathrm{n}^{-}}+(-)^{n} \eta_{\alpha \mu \nu k}^{\bar{\sigma} *} \rho_{\mathrm{n}^{-}} a_{\nu}^{\sigma} \\
\check{\mathcal{C}}_{\mathbf{m}} \rho_{\check{\mathrm{n}}^{-}} & \equiv \sum_{\nu} \check{\eta}_{\alpha \mu \nu m}^{\sigma}\left[a_{\nu}^{\sigma} \rho_{\check{\mathrm{n}}^{-}}-(-)^{n} \rho_{\check{\mathrm{n}}^{-}} a_{\nu}^{\sigma}\right]
\end{aligned}
$$

The tier-up term in Eq. (8.6) arises from the contribution of $\partial_{t} \mathcal{F}=-\mathcal{R} \mathcal{F}$ to $\partial_{t} \mathcal{F}_{\mathrm{n}}$. It is given by

$$
\rho_{\mathrm{n}}^{\{+\}}=-i \sum_{\mathbf{k}}(-)^{q} \mathcal{A}_{\mu}^{\bar{\sigma}} \rho_{\mathrm{n}_{\mathbf{k}}^{+}}-i \sum_{\mathbf{m}} \mathcal{A}_{\mu}^{\bar{\sigma}} \rho_{\check{\mathrm{n}}_{\mathbf{m}}^{+}},
$$

with the $(n+1)^{\text {th }}$-tier of ADOs of [cf. Eq. (C10)]

$$
\begin{aligned}
\rho_{\mathrm{n}_{\mathbf{k}}^{+}} & \equiv \rho_{\mathbf{k}_{1} \cdots \mathbf{k}_{p} \mathbf{k}, \mathbf{m}_{1} \cdots \mathbf{m}_{q}}^{(n+1)}, \\
\rho_{\check{n}_{\mathbf{m}}^{+}} & \equiv \rho_{\mathbf{k}_{1} \cdots \mathbf{k}_{p}, \mathbf{m}_{1} \cdots \mathbf{m}_{q} \mathbf{m}}^{(n+1)}
\end{aligned}
$$

The Grassmann parity associated Liouville-space operator $\mathcal{A}_{\mu}^{\bar{\sigma}}$ in Eq. (8.13) are given by [cf. Eq. (4.15a)]

$$
\mathcal{A}_{\mu}^{\bar{\sigma}} \rho_{\mathrm{n}^{+}}=a_{\mu}^{\bar{\sigma}} \rho_{\mathrm{n}^{+}}-(-)^{n} \rho_{\mathrm{n}^{+}} a_{\mu}^{\bar{\sigma}} .
$$

The signs such as that $(-)^{n-j}$ and $(-)^{q-l}$ in Eq. (8.10) and $(-)^{q}$ in Eq. (8.13) result from the required timeordering rearrangements, together with the Grassmann anticommutation relation; see Appendix C for details.

The $n$ indexes in $\rho_{\mathrm{n}}$, as specified in Eq. (8.7), should all be distinct, due to the Grassmann anticommutation relation. As results, the hierarchy in Eq. (8.6) would be finite, provided that the exponential series of reservoir correlation functions [Eq. (8.2)] is effectively finite, such as the high-temperature cases. At zero temperature, the number of Matsubara terms required goes to infinity, and the HEOM via the present parametrization scheme fails. Nevertheless, the MFD-HEOM formalism in Eq. (5.1) is remains valid, despite the cost due to the multi-dimensional frequency integration. Like its frequency-dispersed counterpart, the $n^{\text {th }}$-tier ADO, $\rho_{\mathrm{n}}$, is of the $(2 n)^{\mathrm{th}}-$ order system-reservoir coupling for its leading term. The hierarchy truncation can therefore be done in a similar manner, such as setting all $\rho^{\left(n>N_{\text {trun }}\right)} \approx 0$, followed by a convergency test. Apparently, the transport current can be readily expressed in terms of the first-tier ADO [cf. Eq. (3.13)].

\section{CONCLUDING REMARKS}

We have established the HEOM formalism, via both the MFD and the parametrization schemes (Sec. [V] and Sec.VIII), for the dynamics of a general electron/spin system in contact with electrodes. It provides a unified tool to the study of a variety of quantum transport behaviors. These include the effects of Coulomb interaction, time-dependent electric potentials (external fields) applied on electrodes (system), multiple-terminals with different temperatures, and non-Markovian reservoir couplings on transport current.

It is easy to show that the commonly used secondorder QDT can be recovered with the first-tier truncation, while various fourth-order theories, such as the Liouville equation in Ref. 49 are of the second-tier approximation here. In particular, we have demonstrated explicitly that the real-time diagrammatic formalism $8,9,10,11,12$ 
that has been used in the study of Kondo physics in quantum transport systems amounts to the second-tier truncation of the present HEOM formalism; see Sec. VII

The present theory is in principle exact, as the only approximation involved, the initial factorization ansatz in Eq. (3.1), can be removed by setting the initial time $t_{0}$ to infinite past. Therefore, at any given finite time before the application of time-dependent external fields, say $t=0$, the reduced system together with its grand canonical bath environment are in a steady state. This is determined as the steady-state solutions to the MFD-HEOM formalism, Eq. (5.1) for general cases or Eq. (6.6) for single-particle systems, at either equilibrium if $\mu_{\alpha}=\mu_{\alpha}^{\text {eq }}$, or nonequilibrium if $\mu_{\alpha} \neq \mu_{\alpha}^{\mathrm{eq}}$ but time independent. Not only to the reduced system density operator, the steadystate solutions are also to those bath-induced auxiliary ones. They carry all relevant information on the correlations between system and reservoirs, as dictated by the HEOM theory. The resulting stationary solutions are used as the initial conditions at $t=0$. The subsequent reduced dynamics and transient transport properties are then evaluated via the present formalism again, upon switch-on of time-dependent $\Delta_{\alpha}(t)$, in additional to the constant $\mu_{\alpha}$ at earlier time. Consequently, the present hierarchical QDT formalism is exact, without any approximation.

The present theory recovers exactly the LandauerBüttiker's transport current expression, as it should. The resulting RSPDM-HEOM [Eq. (6.6)], which is exact for a single-particle system, is particular appealing due to its numerical feasibility for large systems. It may lead to a practical scheme of the time-dependent density functional theory (TDDFT) for open many-particle systems. In principle, this can be done by combining the present RSPDM-HEOM with the conventional DFT ${ }^{50,51,52}$ It is anticipated that the single-particle $h$-matrix in Eq. 6.6. be mapped to the Kohn-Sham counterpart, 22,53,54,55

$$
h_{\mu \nu}(t)=h_{\mu \nu}^{0}+v_{\mu \nu}^{\mathrm{xc}}(t)+\sum_{\mu^{\prime} \nu^{\prime}} \varrho_{\mu^{\prime} \nu^{\prime}}(t) V_{\mu \nu \mu^{\prime} \nu^{\prime}}
$$

where $h^{0}$ is the single-electron contribution and $V_{\mu \nu \mu^{\prime} \nu^{\prime}}$ the two-electron Coulomb integral. The key issue is how to identify the exchange--correlation potential $v_{\mu \nu}^{\mathrm{xc}}(t)$ in the TDDFT for open many-particle systems. The exact HEOM formalism, which is numerically feasible for model Coulomb-interaction systems, may shed some light on the construction of exchange-correlation functionals.

\section{Acknowledgments}

Support from the RGC (604007) of Hong Kong is acknowledged.

\section{APPENDIX A: PATH INTEGRAL FORMALISM: DERIVATION}

The formal solution to the total density operator in the $h_{\mathrm{B}}$-interaction is

$$
\rho_{\mathrm{T}}(t)=U_{\mathrm{T}}\left(t, t_{0} ;\left\{\hat{f}_{\alpha \mu}^{\sigma}(t)\right\}\right) \rho_{\mathrm{T}}\left(t_{0}\right) U_{\mathrm{T}}^{\dagger}\left(t, t_{0} ;\left\{\hat{f}_{\alpha \mu}^{\sigma}(t)\right\}\right),
$$

with $U_{\mathrm{T}}\left(t, t_{0} ;\left\{\hat{f}_{\alpha \mu}^{\sigma}(t)\right\}\right)$ being the stochastic Hilbertspace propagator, satisfying $\partial_{t} U_{\mathrm{T}}=-i\left[H+H^{\prime}(t)\right] U_{\mathrm{T}}$. Let $\{|\psi\rangle\}$ be a second-quantization basis set in the system subspace. The PI expression of $U_{\mathrm{T}}$ reads

$$
\begin{gathered}
U_{\mathrm{T}}\left(\psi, t ; \psi_{0}, t_{0} ;\left\{\hat{f}_{\alpha \mu}^{\sigma}(t)\right\}\right) \\
=\int_{\psi_{0}}^{\psi} \mathcal{D} \psi e^{i S[\psi]} \exp _{+}\left\{-i \sum_{\alpha \mu} \int_{t_{0}}^{t} d \tau\left(a_{\mu}[\psi(\tau)] \hat{f}_{\alpha \mu}^{\dagger}(\tau)\right.\right. \\
\left.\left.+\hat{f}_{\alpha \mu}(\tau) a_{\mu}^{\dagger}[\psi(\tau)]\right)\right\}
\end{gathered}
$$

The action functional $S[\psi]$ is related to the isolated system Hamiltonian only; $\left\{a_{\mu}^{\sigma}[\psi(\tau)]\right\}$ are the Grassmann variables, $, 42,43$ as they denote the creation/annihilation operator of system in the Fermion field PI representation. On the other hand, the stochastic bath variables $\left\{\hat{f}_{\alpha \mu}^{\sigma}(t)\right\}$ remain as the original operators, for which the time-ordered exponential function is needed.

Consider now the reduced system density matrix $\rho(t) \equiv \operatorname{tr}_{\mathrm{B}}\left[\rho_{\mathrm{T}}(t)\right]$. Using Eq. (A1), together with the initial factorization ansatz of Eq. 3.1), it is obtained that (setting $\beta=\beta_{\alpha}$ for simplicity)

$$
\begin{aligned}
& \rho(t)= \operatorname{tr}_{\mathrm{B}}\left[U_{\mathrm{T}}\left(t, t_{0} ;\left\{\hat{f}_{\alpha \mu}^{\sigma}(t)\right\}\right) \rho_{\mathrm{T}}\left(t_{0}\right) U_{\mathrm{T}}^{\dagger}\left(t, t_{0} ;\left\{\hat{f}_{\alpha \mu}^{\sigma}(t)\right\}\right)\right] \\
&=\operatorname{tr}_{\mathrm{B}}\left[e^{\beta\left(h_{\mathrm{B}}-\boldsymbol{\mu} \hat{\boldsymbol{N}}\right)} U_{\mathrm{T}}\left(t, t_{0} ;\left\{\hat{f}_{\alpha \mu}^{\sigma}(t)\right\}\right) e^{-\beta\left(h_{\mathrm{B}}-\boldsymbol{\mu} \hat{\boldsymbol{N}}\right)}\right. \\
&\left.\quad \times \rho\left(t_{0}\right) U_{\mathrm{T}}^{\dagger}\left(t, t_{0} ;\left\{\hat{f}_{\alpha \mu}^{\sigma}(t)\right\}\right) \rho_{\mathrm{B}}^{0}\right] \\
&=\left\langle U_{\mathrm{T}}(t,\right.\left.t_{0} ;\left\{\tilde{f}_{\alpha \mu}^{\sigma}(t-i \beta)\right\}\right) \\
&\left.\times \rho\left(t_{0}\right) U_{\mathrm{T}}^{\dagger}\left(t, t_{0} ;\left\{\hat{f}_{\alpha \mu}^{\sigma}(t)\right\}\right)\right\rangle_{\mathrm{B}},
\end{aligned}
$$

with $\boldsymbol{\mu} \hat{\boldsymbol{N}} \equiv \sum_{\alpha} \mu_{\alpha} \hat{N}_{\alpha}$ and

$$
\begin{aligned}
\tilde{f}_{\alpha \mu}^{\sigma}(t-i \beta) & \equiv e^{\beta\left(h_{\mathrm{B}}-\boldsymbol{\mu} \hat{\mathbf{N}}\right)} \hat{f}_{\alpha \mu}^{\sigma}(t) e^{-\beta\left(h_{\mathrm{B}}-\boldsymbol{\mu} \hat{\mathbf{N}}\right)} \\
& =e^{-\sigma \beta \mu_{\alpha}} \hat{f}_{\alpha \mu}^{\sigma}(t-i \beta)
\end{aligned}
$$

In writing the last identity, the relations of $\left[\hat{N}_{\alpha}, h_{\mathrm{B}}\right]=0$ and $e^{-\beta \boldsymbol{\mu} \hat{\boldsymbol{N}}} f_{\alpha \mu}^{\sigma} e^{\beta \boldsymbol{\mu} \hat{\mathbf{N}}}=e^{-\sigma \beta \mu_{\alpha}} f_{\alpha \mu}^{\sigma}$ are used,, 36 together with Eq. (2.5).

The influence functional used in Eq. (3.2) can then be evaluated by using Eqs. (A2) and A3 together with the Gaussian statistics for the stochastic bath operators 
$\left\{\hat{f}_{\alpha \mu}(t)\right\}$. The details are as follows.

$$
\begin{array}{r}
\mathcal{F}=\left\langle\operatorname { e x p } _ { + } \left\{-i \sum_{\alpha \mu} \int_{t_{0}}^{t} d \tau\left(e^{-\beta \mu_{\alpha}} \hat{f}_{\alpha \mu}^{\dagger}(\tau-i \beta) a_{\mu}[\psi(\tau)]\right.\right.\right. \\
\left.\left.+e^{\beta \mu_{\alpha}} a_{\mu}^{\dagger}[\psi(\tau)] \hat{f}_{\alpha \mu}(\tau-i \beta)\right)\right\} \\
\times \exp _{-}\left\{i \sum _ { \alpha \mu } \int _ { t _ { 0 } } ^ { t } d \tau \left(\hat{f}_{\alpha \mu}^{\dagger}(\tau) a_{\mu}\left[\psi^{\prime}(\tau)\right]\right.\right. \\
\left.\left.\left.+a_{\mu}^{\dagger}\left[\psi^{\prime}(\tau)\right] \hat{f}_{\alpha \mu}(\tau)\right)\right\}\right\rangle_{\mathrm{B}} .
\end{array}
$$

For $\left\{\hat{f}_{\alpha \mu}^{\sigma}(t)\right\}$ satisfying Gaussian statistics, the bath ensemble average in Eq. (A4) can be evaluated exactly by using the second-order cumulant expansion method, as the higher order cumulants are all zero. This property, together with Eq. (2.7), leads to the influence exponent in $\mathcal{F} \equiv \exp (-\Phi)$ the following expression,

$$
\begin{gathered}
\Phi\left[\psi, \psi^{\prime}\right] \\
=\sum_{\alpha \mu \nu} \int_{t_{0}}^{t} d \tau_{2} \int_{t_{0}}^{\tau_{2}} d \tau_{1}\left\{a_{\mu}\left[\psi\left(\tau_{2}\right)\right] a_{\nu}^{\dagger}\left[\psi\left(\tau_{1}\right)\right] C_{\alpha \mu \nu}^{+}\left(\tau_{2}-\tau_{1}\right)\right. \\
+a_{\mu}^{\dagger}\left[\psi\left(\tau_{2}\right)\right] a_{\nu}\left[\psi\left(\tau_{1}\right)\right] C_{\alpha \mu \nu}^{-}\left(\tau_{2}-\tau_{1}\right) \\
\quad+a_{\nu}\left[\psi^{\prime}\left(\tau_{1}\right)\right] a_{\mu}^{\dagger}\left[\psi^{\prime}\left(\tau_{2}\right)\right] C_{\alpha \mu \nu}^{+*}\left(\tau_{2}-\tau_{1}\right) \\
\left.\quad+a_{\nu}^{\dagger}\left[\psi^{\prime}\left(\tau_{1}\right)\right] a_{\mu}\left[\psi^{\prime}\left(\tau_{2}\right)\right] C_{\alpha \mu \nu}^{-*}\left(\tau_{2}-\tau_{1}\right)\right\} \\
-\sum_{\alpha \mu \nu} \int_{t_{0}}^{t} d \tau_{2} \int_{t_{0}}^{t} d \tau_{1}\left\{a_{\mu}\left[\psi\left(\tau_{2}\right)\right] a_{\nu}^{\dagger}\left[\psi^{\prime}\left(\tau_{1}\right)\right] C_{\alpha \mu \nu}^{-*}\left(\tau_{2}-\tau_{1}\right)\right. \\
\left.\quad+a_{\mu}^{\dagger}\left[\psi\left(\tau_{2}\right)\right] a_{\nu}\left[\psi^{\prime}\left(\tau_{1}\right)\right] C_{\alpha \mu \nu}^{+*}\left(\tau_{2}-\tau_{1}\right)\right\}
\end{gathered}
$$

Here, we have used the symmetry relation [the first identity of Eq. (2.7)] in the third and fourth terms, and the detailed-balance relation [the second identity of Eq. (2.7)] in the last two terms of the above expression. Some elementary algebra will then lead to Eq. (A5), recast in terms of $\mathcal{R} \equiv \partial_{t} \Phi$, the expression,

$$
\begin{gathered}
\mathcal{R}[t ;\{\boldsymbol{\psi}\}]=\sum_{\alpha \mu \sigma}\left(a_{\mu}^{\bar{\sigma}}[\psi(t)]\left\{B_{\alpha \mu}^{\sigma}(t ;\{\psi\})-B_{\alpha \mu}^{\prime \sigma}\left(t ;\left\{\psi^{\prime}\right\}\right)\right\}\right. \\
\left.-\left\{B_{\alpha \mu}^{\sigma}(t ;\{\psi\})-B_{\alpha \mu}^{\prime \sigma}\left(t ;\left\{\psi^{\prime}\right\}\right)\right\} a_{\mu}^{\bar{\sigma}}\left[\psi^{\prime}(t)\right]\right) \\
=i \sum_{\alpha \mu \sigma}\left\{a_{\mu}^{\bar{\sigma}}[\psi(t)] \mathcal{B}_{\alpha \mu}^{\sigma}(t ;\{\boldsymbol{\psi}\})\right. \\
\left.-\mathcal{B}_{\alpha \mu}^{\sigma}(t ;\{\boldsymbol{\psi}\}) a_{\mu}^{\bar{\sigma}}\left[\psi^{\prime}(t)\right]\right\} .
\end{gathered}
$$

Here, $\mathcal{B}_{\mu}^{\sigma}$ and $B_{\mu}^{\sigma}\left(B_{\mu}^{\prime \sigma}\right)$ are the same Grassmann variables defined in Eq. (3.5) and Eq. (3.6), respectively. In particular, $\mathcal{B}_{\alpha \mu}^{\sigma} a_{\mu}^{\bar{\sigma}}\left[\psi^{\prime}(t)\right]=-a_{\mu}^{\bar{\sigma}}\left[\psi^{\prime}(t)\right] \mathcal{B}_{\alpha \mu}^{\sigma}$, since $a_{\mu}^{\bar{\sigma}}\left[\psi^{\prime}(t)\right]$ is also a Grassmann variable. With $\mathcal{A}_{\mu}^{\sigma}$ defined in Eq. (3.4), the above equation is identical to Eq. (3.3).

\section{APPENDIX B: DERIVATION OF EOM (6.6) FOR SINGLE-PARTICLE HAMILTONIAN SYSTEMS}

Applying the primary-tier MFD-HEOM, i.e., Eq. (5.1) with $n=0$, for the RSPDM of Eq. (6.2) leads to

$$
\begin{aligned}
i \dot{\varrho}_{\mu \nu}(t)= & \operatorname{tr}_{\mathrm{s}}\left[\left(a_{\nu}^{\dagger} a_{\mu} \mathcal{L}\right) \rho(t)\right] \\
& +\sum_{\alpha m \sigma} \int d \omega \operatorname{tr}_{\mathrm{s}}\left[\left(a_{\nu}^{\dagger} a_{\mu} \mathcal{A}_{m}^{\bar{\sigma}}\right) \phi_{\alpha m}^{\sigma}(\omega, t)\right] \\
= & \operatorname{tr}_{\mathrm{s}}\left\{\left[a_{\nu}^{\dagger} a_{\mu}, H\right] \rho(t)\right\} \\
& +\sum_{\alpha m \sigma} \int d \omega \operatorname{tr}_{\mathrm{s}}\left\{\left[a_{\nu}^{\dagger} a_{\mu}, a_{m}^{\bar{\sigma}}\right] \phi_{\alpha m}^{\sigma}(\omega, t)\right\} .
\end{aligned}
$$

The second identity arises from the trace cyclic invariance. For the single-particle system of Eq. (6.1),

$$
\left[a_{\nu}^{\dagger} a_{\mu}, H\right]=\sum_{m}\left(h_{\mu m} a_{\nu}^{\dagger} a_{m}-h_{m \nu} a_{m}^{\dagger} a_{\nu}\right) .
$$

It leads to

$$
\operatorname{tr}_{\mathrm{s}}\left[\left(a_{\nu}^{\dagger} a_{\mu} \mathcal{L}\right) \rho(t)\right]=[h, \varrho]_{\mu \nu} .
$$

Next, $\left[a_{\nu}^{\dagger} a_{\mu}, a_{m}\right]=-a_{\mu} \delta_{\nu m}$ and $\left[a_{\nu}^{\dagger} a_{\mu}, a_{m}^{\dagger}\right]=a_{\nu}^{\dagger} \delta_{\mu m}$ lead to

$$
\begin{aligned}
& \sum_{\sigma m} \operatorname{tr}_{\mathrm{s}}\left\{\left[a_{\nu}^{\dagger} a_{\mu}, a_{m}^{\bar{\sigma}}\right] \phi_{\alpha m}^{\sigma}(\omega, t)\right\} \\
= & -\operatorname{tr}_{\mathrm{s}}\left[a_{\mu} \phi_{\alpha \nu}^{+}(\omega, t)\right]+\operatorname{tr}_{\mathrm{s}}\left[a_{\nu}^{\dagger} \phi_{\alpha \mu}^{-}(\omega, t)\right] \\
\equiv & -\varphi_{\alpha \mu \nu}^{+}(\omega, t)+\varphi_{\alpha \nu \mu}^{-}(\omega, t),
\end{aligned}
$$

with

$$
\begin{aligned}
& \varphi_{\alpha \mu \nu}^{+}(\omega, t) \equiv \operatorname{tr}_{\mathrm{s}}\left[a_{\mu} \phi_{\alpha \nu}^{+}(\omega, t)\right]=\varphi_{\alpha \mu \nu}(\omega, t), \\
& \varphi_{\alpha \nu \mu}^{-}(\omega, t) \equiv \operatorname{tr}_{\mathrm{s}}\left[a_{\nu}^{\dagger} \phi_{\alpha \mu}^{-}(\omega, t)\right]=\varphi_{\alpha \nu \mu}^{*}(\omega, t) .
\end{aligned}
$$

The second identity in Eq. (B5a) is the same as Eq. (6.3). In writing Eq. (B5b), the property of $\phi_{\alpha \mu}^{\sigma}=\left[\phi_{\alpha \mu}^{\bar{\sigma}}\right]^{\dagger}$ that leads to $\varphi_{\alpha}^{\bar{\sigma}}(\omega, t)=\left[\varphi_{\alpha}^{\sigma}(\omega, t)\right]^{\dagger}$ is used. We have thus arrived at Eq. 6.6a), which is just the matrix form of Eq. (B1).

We are now in the position to show Eq. 6.6b). The MFD-HEOM [Eq. (5.1)] for the first-tier auxiliary RSPDM in Eq. (6.3) reads

$$
\begin{aligned}
i \dot{\varphi}_{\alpha \mu \nu}= & \operatorname{tr}_{\mathrm{s}}\left[\left(a_{\mu} \mathcal{L}\right) \phi_{\alpha \nu}^{+}\right]-\left(\omega+\Delta_{\alpha}\right) \varphi_{\alpha \mu \nu} \\
& +\operatorname{tr}_{\mathrm{s}}\left\{\left[a_{\mu} \mathcal{C}_{\alpha \nu}^{+}(\omega)\right] \rho\right\} \\
& +\int d \omega^{\prime} \sum_{\alpha^{\prime}} \varphi_{\alpha^{\prime} \mu, \alpha \nu}\left(\omega^{\prime}, \omega, t\right),
\end{aligned}
$$

with

$$
\varphi_{\alpha^{\prime} \mu, \alpha \nu} \equiv \sum_{\sigma, m} \operatorname{tr}_{\mathrm{s}}\left[\left(a_{\mu} \mathcal{A}_{m}^{\bar{\sigma}}\right) \phi_{\alpha \nu, \alpha^{\prime} m}^{+, \sigma}\left(\omega, \omega^{\prime}, t\right)\right] .
$$

The first term in the rhs of Eq. (B6) can be evaluated by using the identity, $a_{\mu} \mathcal{L}=\left[a_{\mu}, H\right]=\sum_{m} h_{\mu m} a_{m}$, for the 
present single-particle system, together with Eq. (6.3). It results in

$$
\operatorname{tr}_{\mathrm{s}}\left[\left(a_{\mu} \mathcal{L}\right) \phi_{\alpha \nu}^{+}\right]=\sum_{m}\left(h_{\mu m} \operatorname{tr}_{\mathrm{s}}\left[a_{m} \phi_{\alpha \nu}^{+}\right]\right)=\left(h \varphi_{\alpha}\right)_{\mu \nu}
$$

The third term in the rhs of Eq. (B6) can be evaluated as [cf. Eqs. (5.3b) and (6.3)]

$$
\begin{aligned}
& \operatorname{tr}_{\mathrm{S}}\left\{\left[a_{\mu} \mathcal{C}_{\alpha \nu}^{+}(\omega)\right] \rho\right\} \\
= & \sum_{m} \operatorname{tr}_{\mathrm{s}}\left[\Gamma_{\alpha \nu m}^{+}(\omega) a_{\mu} a_{m}^{\dagger} \rho-\Gamma_{\alpha m \nu}^{-}(\omega) a_{m}^{\dagger} a_{\mu} \rho\right] \\
= & \sum_{m} \Gamma_{\alpha \nu m}^{+}(\omega) \bar{\varrho}_{\mu m}-\Gamma_{\alpha m \nu}^{-}(\omega) \varrho_{\mu m} .
\end{aligned}
$$

Here, $\bar{\varrho}_{\mu m} \equiv \operatorname{tr}_{\mathrm{s}}\left(a_{\mu} a_{m}^{\dagger} \rho\right)=\delta_{\mu m}-\varrho_{\mu m}$; i.e., the elements of reduced single-hole density matrix of $\bar{\varrho} \equiv \mathbf{1}-\varrho$. Together with Eqs. (2.12) and (2.13a), the above equation can be recast as

$$
\operatorname{tr}_{\mathrm{s}}\left\{\left[a_{\mu} \mathcal{C}_{\alpha \nu}^{+}(\omega)\right] \rho\right\}=\left[f_{\alpha}(\omega) J_{\alpha}(\omega)-\varrho J_{\alpha}(\omega)\right]_{\mu \nu} .
$$

Note that due to the Grassmann parity of Eq. (5.3a), $\mathcal{A}_{m}^{\bar{\sigma}}$ in Eq. (B1) behaves as a commutator, its action in Eq. (B7) is an anticommutator. The fact that $\varphi_{\alpha^{\prime} \mu, \alpha \nu}$ defined in Eq. (B7) is identical to the matrix element $\left[\varphi_{\alpha^{\prime} \alpha}\left(\omega^{\prime}, \omega, t\right)\right]_{\mu \nu}$ of Eq. 6.4) can then be readily concluded. We have thus completed Eq. 6.6b), with the $S_{\alpha}(\omega)$ defined in Eq. (6.7).

Finally, Eq. 6.6c) can be readily obtained via the trace of the second-tier MFD-HEOM [Eq. (5.1)], together with Eqs. (2.12), (B5), and the elementary algebra just described.

Note that the coupled EOM, Eq. (6.6), can formally be recast in the standard form as

$$
i \frac{\partial}{\partial t}\left[\begin{array}{l}
\mathrm{X} \\
\mathrm{Y}
\end{array}\right]=[\Lambda+\delta \Lambda(t)]\left[\begin{array}{l}
\mathrm{X} \\
\mathrm{Y}
\end{array}\right]+\left[\begin{array}{l}
0 \\
\mathrm{~S}
\end{array}\right]
$$

with

$$
\mathrm{X} \equiv\left[\begin{array}{c}
\varrho(t) \\
\boldsymbol{\varphi}_{\alpha^{\prime} \alpha}\left(\omega^{\prime}, \omega, t\right)
\end{array}\right] ; \quad \mathrm{Y} \equiv\left[\begin{array}{c}
\boldsymbol{\varphi}_{\alpha}(\omega, t) \\
\boldsymbol{\varphi}_{\alpha^{\prime}}^{\dagger}\left(\omega^{\prime}, t\right)
\end{array}\right]
$$

and

$$
\mathrm{S} \equiv\left[\begin{array}{c}
\boldsymbol{S}_{\alpha}(\omega) \\
-\boldsymbol{S}_{\alpha^{\prime}}\left(\omega^{\prime}\right)
\end{array}\right]
$$

The $4 \times 4$ matrix $\delta \Lambda(t)$ in Eq. (B10a) arises from the time-dependent bias potential. It is diagonal, with the elements of

$$
\delta \Lambda(t)=\left\{0,-\Delta_{\alpha}(t)+\Delta_{\alpha^{\prime}}(t),-\Delta_{\alpha}(t), \Delta_{\alpha^{\prime}}(t)\right\} .
$$

The time-independent counterpart is given in terms of block-matrix form as

$$
\Lambda=\left[\begin{array}{ll}
\Lambda_{\mathrm{xx}} & \Lambda_{\mathrm{xy}} \\
\Lambda_{\mathrm{yx}} & \Lambda_{\mathrm{yy}}
\end{array}\right]
$$

with

$$
\begin{array}{ll}
\Lambda_{\mathrm{xx}}=\left[\begin{array}{cc}
\overleftrightarrow{h} & 0 \\
0 & \omega^{\prime}-\omega
\end{array}\right], \quad \Lambda_{\mathrm{xy}}=\left[\begin{array}{cc}
-\mathbf{1}_{\alpha} & \mathbf{1}_{\alpha^{\prime}} \\
\vec{J}_{\alpha^{\prime}} & -\overleftarrow{J}_{\alpha}
\end{array}\right], \\
\Lambda_{\mathrm{yx}}=\left[\begin{array}{cc}
-\overleftarrow{J}_{\alpha} & \mathbf{1}_{\alpha^{\prime}} \\
\vec{J}_{\alpha^{\prime}} & -\mathbf{1}_{\alpha}
\end{array}\right], \quad \Lambda_{\mathrm{yy}}=\left[\begin{array}{cc}
\vec{h}-\omega & 0 \\
0 & \omega^{\prime}-\overleftarrow{h}
\end{array}\right],
\end{array}
$$

where

$$
\mathbf{1}_{\alpha} \equiv \sum_{\alpha} \int d \omega, \quad \mathbf{1}_{\alpha^{\prime}} \equiv \sum_{\alpha^{\prime}} \int d \omega^{\prime}
$$

Introduced in Eq. (B12) is also the tetradic notation for the left- or right-multiplication action of a matrix $O$ on the matrix $A$ of interest as

$$
\vec{O} A \equiv O A, \quad \overleftarrow{O} A \equiv A O^{\dagger}, \text { and } \stackrel{\leftrightarrow}{O} \equiv \vec{O}-\overleftarrow{O}
$$

In other words, $\vec{O}$ and $\overleftarrow{O}$ are tensors, with the elements of $\vec{O}_{m n, m^{\prime} n^{\prime}}=O_{m m^{\prime}} \delta_{n^{\prime} n}$ and $\overleftarrow{O}_{m n, m^{\prime} n^{\prime}}=\delta_{m m^{\prime}} O_{n n^{\prime}}^{*}$ Implied in Eq. (B12) is also the frequency variable associating with the subindex; i.e., $\overleftarrow{J}_{\alpha} \equiv \overleftarrow{J}_{\alpha}(\omega)$ and $\vec{J}_{\alpha^{\prime}} \equiv \vec{J}_{\alpha^{\prime}}\left(\omega^{\prime}\right)$

\section{APPENDIX C: DERIVATION OF HEOM (8.6)}

Denote $\mathbf{k} \equiv(\alpha \mu \nu \sigma k)$ and $\mathbf{m} \equiv(\alpha \mu \sigma m)$ for short, and introduce

$$
\begin{aligned}
B_{\mathbf{k}}(t ;\{\psi\}) & \equiv \int_{t_{0}}^{t} d \tau e^{-\theta_{\mathbf{k}}(t, \tau)} a_{\nu}^{\sigma}[\psi(\tau)], \\
\check{B}_{\mathbf{m}}(t ;\{\psi\}) & \equiv \sum_{\nu} \check{\eta}_{\alpha \mu \nu m}^{\sigma} \int_{t_{0}}^{t} d \tau e^{-\check{\theta}_{\alpha m}^{\sigma}(t, \tau)} a_{\nu}^{\sigma}[\psi(\tau)],
\end{aligned}
$$

with $\theta_{\mathbf{k}}(t, t)=\check{\theta}_{\alpha m}^{\sigma}(t, t)=0$, and

$$
\begin{aligned}
\partial_{t} \theta_{\mathbf{k}}(t, \tau) & =\gamma_{\alpha \mu \nu}^{\sigma}-\sigma i \Delta_{\alpha}(t), \\
\partial_{t} \check{\theta}_{\alpha m}^{\sigma}(t, \tau) & =\check{\gamma}_{\alpha m}^{\sigma}-\sigma i \Delta_{\alpha}(t) .
\end{aligned}
$$

The two functionals in Eq. (C1) are the counterparts of that in Eq. (3.6a), arising from the two distinct components of reservoir correlation functions as Eq. (8.2). The nonstationary exponential factor in Eq. (2.16), due to time-dependent chemical potentials applied to electrodes, are also accounted for via the nonstationary phases as Eq. (C2).

The dissipation functional [Eq. (3.3b)] is now decomposed according to the parametrization of Eq. (8.2) as

$$
\mathcal{R}=i \sum_{\mathbf{k}} \mathcal{A}_{\mu}^{\bar{\sigma}} \mathcal{B}_{\mathbf{k}}+i \sum_{\mathbf{m}} \mathcal{A}_{\mu}^{\bar{\sigma}} \check{\mathcal{B}}_{\mathbf{m}}
$$

which is the same as Eq. (8.5), with [cf. Eq. (3.5)],

$$
\begin{aligned}
\mathcal{B}_{\mathbf{k}} & \equiv-i\left[\eta_{\alpha \mu \nu k}^{\sigma} B_{\mathbf{k}}(t ;\{\psi\})-\eta_{\alpha \mu \nu k}^{\bar{\sigma} *} B_{\mathbf{k}}\left(t ;\left\{\psi^{\prime}\right\}\right)\right], \\
\check{\mathcal{B}}_{\mathbf{m}} & \equiv-i\left[\check{B}_{\mathbf{m}}(t ;\{\psi\})+\check{B}_{\mathbf{m}}\left(t ;\left\{\psi^{\prime}\right\}\right)\right] .
\end{aligned}
$$


In writing Eq. (C4b), the last relation in Eq. (8.4b) is applied. The time derivatives on Eq. (C4) can be obtained as [cf. Eq. (C2)]

$$
\begin{aligned}
\partial_{t} \mathcal{B}_{\mathbf{k}} & =-\left[\gamma_{\alpha \mu \nu k}^{\sigma}-\sigma i \Delta_{\alpha}(t)\right] \mathcal{B}_{\mathbf{k}}-i \mathcal{C}_{\mathbf{k}}, \\
\partial_{t} \check{\mathcal{B}}_{\mathbf{m}} & =-\left[\check{\gamma}_{\alpha m}^{\sigma}-\sigma i \Delta_{\alpha}(t)\right] \check{\mathcal{B}}_{\mathbf{m}}-i \check{\mathcal{C}}_{\mathbf{m}}
\end{aligned}
$$

with

$$
\begin{aligned}
\mathcal{C}_{\mathbf{k}} & =\eta_{\alpha \mu \nu k}^{\sigma} a_{\nu}^{\sigma}[\psi(t)]-\eta_{\alpha \mu \nu k}^{\bar{\sigma} *} a_{\nu}^{\sigma}\left[\psi^{\prime}(t)\right], \\
\check{\mathcal{C}}_{\mathbf{m}} & =\sum_{\nu} \check{\eta}_{\alpha \mu \nu m}^{\sigma}\left(a_{\nu}^{\sigma}[\psi(t)]+a_{\nu}^{\sigma}\left[\psi^{\prime}(t)\right]\right),
\end{aligned}
$$

in the PI representation.

The $n^{\text {th }}$-tier AIFs can in general be defined as

$$
\mathcal{F}_{\mathbf{n}} \equiv \mathcal{F}_{\mathbf{k}_{1} \cdots \mathbf{k}_{p}, \mathbf{m}_{1} \cdots \mathbf{m}_{q}}^{(n)} \equiv \check{\mathcal{B}}_{\mathbf{m}_{q}} \cdots \check{\mathcal{B}}_{\mathbf{m}_{1}} \mathcal{B}_{\mathbf{k}_{p}} \cdots \mathcal{B}_{\mathbf{k}_{1}} \mathcal{F}
$$

with $n \equiv p+q$. The associated $n^{\text {th }}-$ tier ADO $\rho_{\mathrm{n}}$ is defined via (noting that $\rho \equiv \rho_{0}$ )

$$
\rho_{\mathrm{n}}(t) \equiv \mathcal{U}_{\mathrm{n}}\left(t, t_{0}\right) \rho\left(t_{0}\right),
$$

with the propagator,

$$
\mathcal{U}_{\mathbf{n}}\left(t, \boldsymbol{\psi} ; t_{0}, \boldsymbol{\psi}_{0}\right) \equiv \int_{\boldsymbol{\psi}_{0}\left[t_{0}\right]}^{\boldsymbol{\psi}[t]} \mathcal{D} \boldsymbol{\psi} e^{i S[\psi]} \mathcal{F}_{\mathbf{n}} e^{-i S\left[\psi^{\prime}\right]}
$$

in the PI representation.

The HEOM formalism [Eqs. 8.6) -8.14)] for $\rho_{\mathrm{n}}$ can be followed immediately via the time derivative on $\mathcal{F}_{n}$. The latter is carried out by using Eq. (C5), together with $\partial_{t} \mathcal{F}=-\mathcal{R} \mathcal{F}$ and Eq. (C) 3 . Some details are as follows.

The $\gamma_{\mathrm{n}}(t)$ in Eqs. (8.6) and (8.9) arises from the squarebracket terms in the rhs of Eq. (C5). It collects the time derivatives on the nonstationary phases of Eq. (C2).
The second terms in Eq. (C5) leads to the tier-down dependence, as shown by Eq. (8.10), with the involving $(n-1)^{\text {th }}$-tier ADOs of Eq. (8.11) associating with

$$
\begin{aligned}
& \mathcal{F}_{\mathbf{n}_{j}^{-}} \equiv \check{\mathcal{B}}_{\mathbf{m}_{q}} \cdots \check{\mathcal{B}}_{\mathbf{m}_{1}} \mathcal{B}_{\mathbf{k}_{p}} \cdots \mathcal{B}_{\mathbf{k}_{j+1}} \mathcal{B}_{\mathbf{k}_{j-1}} \cdots \mathcal{B}_{\mathbf{k}_{1}} \mathcal{F} \\
& \mathcal{F}_{\check{\mathbf{n}}_{l}^{-}} \equiv \check{\mathcal{B}}_{\mathbf{m}_{q}} \cdots \check{\mathcal{B}}_{\mathbf{m}_{l+1}} \check{\mathcal{B}}_{\mathbf{m}_{l-1}} \cdots \check{\mathcal{B}}_{\mathbf{m}_{1}} \mathcal{B}_{\mathbf{k}_{p}} \cdots \mathcal{B}_{\mathbf{k}_{1}} \mathcal{F}
\end{aligned}
$$

The $\mathcal{C}$-functionals in Eqs. (C5) and (C6), which depend only on the fixed ending points of PI at the local time $t$, are now superoperators in Eqs. (8.10) and (8.12) with Grassmann parity. The signs $(-)^{n-j}$ and $(-)^{q-l}$ in Eq. (8.10) are associated with the time-ordering arrangement. It brings the $\mathcal{C}$ and $\check{\mathcal{C}}$ functionals, originally at the $\mathbf{k}_{j}$ and $\mathbf{m}_{l}$ positions, respectively, to the left most of action, as indicated in Eq. (C9).

The identity of $\partial_{t} \mathcal{F}=-\mathcal{R F}$ with Eq. (C3) contributes to $\partial_{t} \mathcal{F}_{\mathbf{n}}[$ Eq. $[\mathbf{C} 7$ ] $]$ the terms of

$$
\begin{aligned}
\mathcal{A}_{\mu}^{\bar{\sigma}} \mathcal{B}_{\mathbf{k}} \mathcal{F}_{\mathbf{n}} & =(-)^{q} \mathcal{A}_{\mu}^{\bar{\sigma}}\left(\check{\mathcal{B}}_{\mathbf{m}_{q}} \cdots \check{\mathcal{B}}_{\mathbf{m}_{1}} \mathcal{B}_{\mathbf{k}} \mathcal{B}_{\mathbf{k}_{p}} \cdots \mathcal{B}_{\mathbf{k}_{1}} \mathcal{F}\right) \\
& \equiv(-)^{q} \mathcal{A}_{\mu}^{\bar{\sigma}} \mathcal{F}_{\mathbf{n}_{\mathbf{k}}^{+}}, \\
\mathcal{A}_{\mu}^{\bar{\sigma}} \check{\mathcal{B}}_{\mathbf{m}} \mathcal{F}_{\mathbf{n}} & =\mathcal{A}_{\mu}^{\bar{\sigma}}\left(\check{\mathcal{B}}_{\mathbf{m}} \check{\mathcal{B}}_{\mathbf{m}_{q}} \cdots \check{\mathcal{B}}_{\mathbf{m}_{1}} \mathcal{B}_{\mathbf{k}_{p}} \cdots \mathcal{B}_{\mathbf{k}_{1}} \mathcal{F}\right) \\
& \equiv \mathcal{A}_{\mu}^{\bar{\sigma}} \mathcal{F}_{\check{\mathbf{n}}_{\mathbf{m}}^{+}} .
\end{aligned}
$$

They correspond to the two terms in Eq. (8.13), respectively. The involving $(n+1)^{\text {th }}$-tier ADOs, $\rho_{\mathrm{n}_{\mathbf{k}}^{+}}$and $\rho_{\check{n}_{\mathrm{m}}^{+}}$ [Eq. (8.14)], are associated with $\mathcal{F}_{\mathrm{n}_{k}^{+}}$and $\mathcal{F}_{\mathfrak{n}_{m}^{+}}$, respectively, defined in the second identities above. We have thus completed the derivation of the HEOM formalism via parametrization presented in Sec. VIII.
1 S. Datta, Electronic Transport in Mesoscopic Systems, Oxford University Press, New York, 1995.

${ }^{2}$ H. Haug and A.-P. Jauho, Quantum Kinetics in Transport and Optics of Semiconductors, Springer-Verlag, Berlin, 1996, Springer Series in Solid-State Sciences 123.

3 S. Hershfield, J. H. Davies, and J. W. Wilkins, Phys. Rev. Lett. 67, 3720 (1991).

4 A. L. Yeyati, A. Martín-Rodero, and F. Flores, Phys. Rev. Lett. 71, 2991 (1993).

5 T. Fujii and K. Ueda, Phys. Rev. B 68, 155310 (2003).

6 Y. Meir, N. S. Wingreen, and P. A. Lee, Phys. Rev. Lett. 66, 3048 (1991).

7 Y. Meir, N. S. Wingreen, and P. A. Lee, Phys. Rev. Lett. 70, 2601 (1993).

${ }^{8}$ H. Schoeller and G. Schön, Phys. Rev. B 50, 18436 (1994).

9 H. Schoeller and G. Schön, Physica B 203, 423 (1994).

10 J. König, H. Schoeller, and G. Schön, Europhys. Lett. 31, 31 (1995).

11 J. König, J. Schmid, H. Schoeller, and G. Schön, Phys. Rev. B 54, 16820 (1996).
12 J. König, H. Schoeller, and G. Schön, Phys. Rev. Lett. 76, 1715 (1996).

13 K. G. Wilson, Rev. Mod. Phys. 47, 773 (1975).

14 H. R. Krishna-murthy, J. W. Wilkins, and K. G. Wilson, Phys. Rev. B 21, 1044 (1980).

15 A. C. Hewson, The Kondo Problem to Heavy Fermions, Cambridge University Press, Cambridge, 1993.

16 C. Bruder and H. Schoeller, Phys. Rev. Lett. 72, 1076 (1994).

17 P. Brune, C. Bruder, and H. Schoeller, Phys. Rev. B 56, 4730 (1997).

18 J. Lehmann, S. Kohler, P. Hänggi, and A. Nitzan, Phys. Rev. Lett. 88, 228305 (2002).

19 X. Q. Li, J. Y. Luo, Y. G. Yang, P. Cui, and Y. J. Yan, Phys. Rev. B 71, 205304 (2005).

20 S. Welack, M. Schreiber, and U. Kleinekathöfer, J. Chem. Phys. 044712, 124 (2006).

${ }^{21}$ U. Harbola, M. Esposito, and S. Mukamel, Phys. Rev. B 74, 235309 (2006).

${ }^{22}$ X. Q. Li and Y. J. Yan, Phys. Rev. B 75, 075114 (2007). 
${ }^{23}$ P. Cui, X. Q. Li, J. S. Shao, and Y. J. Yan, Phys. Lett. A 357, 449 (2006).

24 S. De Franceschi, S. Sasaki, J. M. Elzerman, W. G. van der Wiel, S. Tarucha, and L. P. Kouwenhoven, Phy. Rev. Lett. 86, 878 (2001).

${ }^{25}$ R. Schleser, T. Ihn, E. Ruh, K. Ensslin, M. Tews, D. Pfannkuche, D. C. Driscoll, and A. C. Gossard, Phys. Rev. Lett. 94, 206805 (2005).

26 S. Nakajima, Prog. Theor. Phys. 20, 948 (1958).

27 R. W. Zwanzig, Statistical Mechanics of Irreversibility: Lectures in Theoretical Physics, volume III, Wiley, New York, 1961.

28 H. Mori, Prog. Theor. Phys. 33, 423 (1965).

29 S. A. Gurvitz, Phys. Rev. B 56, 15215 (1997).

30 X. Q. Li, W. K. Zhang, P. Cui, J. S. Shao, Z. S. Ma, and Y. J. Yan, Phys. Rev. B 69, 085315 (2004).

31 D. Loss and D. P. DiVincenzo, Phys. Rev. A 57, 120 (1998).

32 T. Fujisawa, T. Hayashi, and S. Sasaki, Rep. Prog. Phys 69, 759 (2006).

${ }^{33}$ Y. J. Yan and R. X. Xu, Annu. Rev. Phys. Chem. 56, 187 (2005).

${ }^{34}$ R. X. Xu, P. Cui, X. Q. Li, Y. Mo, and Y. J. Yan, J. Chem. Phys. 122, 041103 (2005).

35 R. X. Xu and Y. J. Yan, Phys. Rev. E 75, 031107 (2007).

36 J. S. Jin, S. Welack, J. Y. Luo, X. Q. Li, P. Cui, R. X. Xu, and Y. J. Yan, J. Chem. Phys. 126, 134113 (2007).

37 Y. Tanimura and R. Kubo, J. Phys. Soc. Jpn. 58, 101 (1989).

38 Y. Tanimura, Phys. Rev. A 41, 6676 (1990).

39 Y. Tanimura, J. Phys. Soc. Jpn. 75, 082001 (2006).
40 U. Weiss, Quantum Dissipative Systems, World Scientific, Singapore, 1999, 2nd ed. Series in Modern Condensed Matter Physics, Vol. 10.

41 R. X. Xu and Y. J. Yan, J. Chem. Phys. 116, 9196 (2002).

42 L. H. Ryder, Quantum Field Theory, Cambridge University Press, Cambridge, 1996, 2nd ed.

${ }^{43}$ H. Kleinert, Path Integrals in Quantum Mechanics, Statistics, Polymer Physics, and Financial Markets, World Scientific, Singapore, 2006, 4th ed.

44 R. P. Feynman and F. L. Vernon, Jr., Ann. Phys. (N. Y.) 24, 118 (1963).

45 C. Meier and D. J. Tannor, J. Chem. Phys. 111, 3365 (1999).

46 Y. Meir and N. S. Wingreen, Phys. Rev. Lett. 68, 2512 (1992).

47 R. Landauer, Physica Scripta T42, 110 (1992).

48 M. Büttiker, IBM J. Res. Dev. 32, 317 (1988).

49 J. N. Pedersen and A. Wacker, Phys. Rev. B 72, 195330 (2005).

50 P. Hohenberg and W. Kohn, Phys. Rev. 136, B864 (1964).

51 W. Kohn and L. J. Sham, Phys. Rev. 140, A1133 (1965).

52 E. Runge and E. K. U. Gross, Phys. Rev. Lett. 52, 997 (1984).

53 C. Y. Yam, S. Yokojima, and G. H. Chen, Phys. Rev. B 68, 153105 (2003).

54 X. Zheng, F. Wang, C. Y. Yam, Y. Mo, and G. H. Chen, Phys. Rev. B 75, 195127 (2007).

55 M. Di Ventra and R. D'Agosta, Phys. Rev. Lett. 98, 226403 (2007). 